\title{
Sloan Digital Sky Survey Standard Star Catalog for Stripe 82: The Dawn of Industrial 1\% Optical Photometry
}

\section{Citation}

Ivezi?, ?eljko, J. Allyn Smith, Gajus Miknaitis, Huan Lin, Douglas Tucker, Robert H. Lupton, James E. Gunn, et al. 2007. "Sloan Digital Sky Survey Standard Star Catalog for Stripe 82: The Dawn of Industrial 1\% Optical Photometry." The Astronomical Journal 134 (3) (July 10): 973-998. doi:10.1086/519976.

\section{Published Version}

doi:10.1086/519976

\section{Permanent link}

http://nrs.harvard.edu/urn-3:HUL.InstRepos:33461896

\section{Terms of Use}

This article was downloaded from Harvard University's DASH repository, and is made available under the terms and conditions applicable to Other Posted Material, as set forth at http:// nrs.harvard.edu/urn-3:HUL.InstRepos:dash.current.terms-of-use\#LAA

\section{Share Your Story}

The Harvard community has made this article openly available.

Please share how this access benefits you. Submit a story. 


\title{
SLOAN DIGITAL SKY SURVEY STANDARD STAR CATALOG FOR STRIPE 82: THE DAWN OF INDUSTRIAL 1\% OPTICAL PHOTOMETRY
}

\author{
Željko Ivezić, ${ }^{1}$ J. Allyn Smith, ${ }^{2}$ Gajus Miknaitis, ${ }^{3}$ Huan Lin, ${ }^{3}$ Douglas Tucker, ${ }^{3}$ Robert H. Lupton, ${ }^{4}$ James E. Gunn, ${ }^{4}$ \\ Gillian R. Knapp, ${ }^{4}$ Michael A. Strauss, ${ }^{4}$ Branimir Sesar, ${ }^{1}$ Mamoru Doi, ${ }^{5}$ Masayuki Tanaka, ${ }^{6}$ Masataka Fukugita, ${ }^{7}$ \\ Jon Holtzman, ${ }^{8}$ Steve Kent, ${ }^{3}$ Brian Yanny, ${ }^{3}$ David Schlegel, ${ }^{9}$ Douglas Finkbeiner, ${ }^{10}$ Nikhil Padmanabhan, ${ }^{9}$ \\ Constance M. Rockosi, ${ }^{11}$ Mario Jurić, ${ }^{12}$ Nicholas Bond, ${ }^{4}$ Brian Lee, ${ }^{9}$ Chris Stoughton, ${ }^{3}$ \\ Sebastian Jester, ${ }^{13}$ Hugh Harris, ${ }^{14}$ Paul Harding, ${ }^{15}$ Heather Morrison, ${ }^{15}$ \\ Jon Brinkmann, ${ }^{16}$ Donald P. Schneider, ${ }^{17}$ And Donald York ${ }^{18}$ \\ Received 2007 February 24; accepted 2007 May 13
}

\begin{abstract}
We describe a standard star catalog constructed using multiple SDSS photometric observations (at least four per band, with a median of 10) in the ugriz system. The catalog includes 1.01 million nonvariable unresolved objects from the equatorial stripe $82\left(\left|\delta_{\mathrm{J} 2000.0}\right|<1.266^{\circ}\right)$ in the right ascension range $20^{\mathrm{h}} 34^{\mathrm{m}}-4^{\mathrm{h}} 00^{\mathrm{m}}$ and with the corresponding $r$-band (approximately Johnson $V$-band) magnitudes in the range 14-22. The distributions of measurements for individual sources demonstrate that the photometric pipeline correctly estimates random photometric errors, which are below $0.01 \mathrm{mag}$ for stars brighter than 19.5, 20.5, 20.5, 20, and $18.5 \mathrm{in} u g r i z$, respectively (about twice as good as for individual SDSS runs). Several independent tests of the internal consistency suggest that the spatial variation of photometric zero points is not larger than $\sim 0.01 \mathrm{mag}(\mathrm{rms})$. In addition to being the largest available data set with optical photometry internally consistent at the $\sim 1 \%$ level, this catalog provides a practical definition of the SDSS photometric system. Using this catalog, we show that photometric zero points for SDSS observing runs can be calibrated within a nominal uncertainty of $2 \%$ even for data obtained through 1 mag thick clouds, and we demonstrate the existence of $\mathrm{He}$ and $\mathrm{H}$ white dwarf sequences using photometric data alone. Based on the properties of this catalog, we conclude that upcoming large-scale optical surveys such as the Large Synoptic Survey Telescope will be capable of delivering robust $1 \%$ photometry for billions of sources.
\end{abstract}

Key words: catalogs — instrumentation: photometers — methods: data analysis — standards — surveys techniques: photometric

Online material: color figures

\section{INTRODUCTION}

Astronomical optical photometric data are usually calibrated using sets of standard stars whose brightness is known from previous work. The most notable modern optical standard star catalogs are the Landolt standards (Landolt 1992) and Stetson standards (Stetson 2000, 2005). Both are reported on the JohnsonKron-Cousins system (Landolt 1983 and references therein). The Landolt catalog provides magnitudes accurate to $\lesssim 1 \%$ in the $U B V R I$ bands for $\sim 500$ stars in the $V$ magnitude range $11.5-$ 16. Stetson has extended Landolt's work to fainter magnitudes and provided the community with $\sim 1 \%-2 \%$ accurate magnitudes in the $B V R I$ bands for $\sim 15,000$ stars in the magnitude range $V \lesssim 20$.

\footnotetext{
${ }^{1}$ Department of Astronomy, University of Washington, Box 351580, Seattle, WA 98195, USA.

2 Department of Physics and Astronomy, Austin Peay State University, Clarksville, TN 37044, USA.

3 Fermi National Accelerator Laboratory, P.O. Box 500, Batavia, IL 60510, USA.

${ }^{4}$ Princeton University Observatory, Princeton, NJ 08544, USA.

5 Institute of Astronomy, University of Tokyo, 2-21-1 Osawa, Mitaka, Tokyo 181-0015, Japan.

${ }^{6}$ Department of Astronomy, Graduate School of Science, University of Tokyo, Hongo 7-3-1, Bunkyo-ku, Tokyo 113-0033, Japan.

${ }^{7}$ Institute for Cosmic Ray Research, University of Tokyo, Kashiwa, Chiba, Japan.

${ }^{8}$ New Mexico State University, 1320 Frenger Street, Box 30001, Las Cruces, NM 88003, USA.

9 Lawrence Berkeley National Laboratory, 1 Cyclotron Road, MS 50R5032, Berkeley, CA 94720, USA.
}

Most stars from both sets are distributed along the celestial equator, which facilitates their use from both hemispheres.

The data obtained by the Sloan Digital Sky Survey (SDSS; York et al. 2000) can be used to extend the work by Landolt and Stetson to even fainter levels and to increase the number of standard stars to over 1 million. In addition, SDSS has designed its own photometric system (ugriz; Fukugita et al. 1996), which is now in use at many observatories worldwide. This widespread use of the ugriz photometric system motivates the construction of a large standard star catalog with $\sim 1 \%$ accuracy. As part of its imaging survey, SDSS has obtained many scans in the so-called stripe 82 region, which is defined by $\left|\delta_{\mathrm{J} 2000.0}\right|<1.266^{\circ}$ and right ascension approximately in the range $20^{\mathrm{h}}-4^{\mathrm{h}}$. These repeated

\footnotetext{
${ }^{10}$ Harvard-Smithsonian Center for Astrophysics, 60 Garden Street, Cambridge, MA 02138, USA

11 University of California, Santa Cruz, 1156 High Street, Santa Cruz, CA 95060, USA

12 Institute for Advanced Study, 1 Einstein Drive, Princeton, NJ 08540, USA.

${ }^{13}$ School of Physics and Astronomy, University of Southampton, Highfield, Southampton SO17 1BJ, UK.

${ }^{14}$ US Naval Observatory, Flagstaff Station, P.O. Box 1149, Flagstaff, AZ 86002, USA.

${ }^{15}$ Department of Astronomy, Case Western Reserve University, Cleveland, OH 44106 , USA.

${ }^{16}$ Apache Point Observatory, 2001 Apache Point Road, P.O. Box 59, Sunspot, NM 88349-0059, USA.

${ }^{17}$ Department of Astronomy and Astrophysics, The Pennsylvania State University, University Park, PA 16802, USA.

18 Astronomy and Astrophysics Center, University of Chicago, 5640 South Ellis Avenue, Chicago, IL 60637, USA.
} 
observations can be averaged to produce more accurate photometry than the nominal $2 \%$ single-scan accuracy (Ivezić et al. 2004a).

The catalog and methods presented here have some similarity to an effort by Padmanabhan et al. (2007), who developed a new calibration algorithm that simultaneously solves for the calibration parameters and relative stellar fluxes using overlapping SDSS observations (the so-called übercalibration method). The algorithm decouples the problem of "relative" calibrations (i.e., producing an internally consistent system) from that of "absolute" calibrations (i.e., typing the internal system to a physical flux scale): the absolute calibration is reduced to determining a few numbers for the entire survey. Here we also decouple relative and absolute calibrations and use overlapping observations. The main difference between their work and this paper is that they are concerned about calibrating the entire SDSS survey $\left(\sim 8500 \mathrm{deg}^{2} ;\right.$ most of the surveyed area has at most two overlapping observations), while we concentrate here on a much smaller area $\left(\sim 300 \mathrm{deg}^{2}\right)$ with an average of 10 overlapping observations. We also determine flat-field corrections (relative to the "standard" survey reductions) using different methods: Padmanabhan et al. minimize errors in relative photometry of multiply observed stars, while we require that the stellar locus remains fixed in multidimensional color space. An advantage of the catalog presented here is better averaging of various photometric errors thanks to a larger number of observations, which comes at the expense of a much smaller cataloged area. It is encouraging that the results of these two complementary approaches agree in the regions of sky common to both catalogs at the claimed level of accuracy $(\sim 1 \%)$.

Additional motivation for the analysis of repeated scans and their impact on photometric accuracy comes from upcoming largescale optical surveys such as the Dark Energy Survey (Flaugher et al. 2006), Pan-STARRS (Kaiser et al. 2002), and the Large Synoptic Survey Telescope (LSST; Tyson 2002). For example, the LSST science requirements document ${ }^{19}$ calls for a photometric system that is internally consistent across the sky at the $1 \%$ level. The SDSS stripe 82 repeated scans can be used to gauge the plausibility of delivering such a system.

We describe the construction and testing of a standard star catalog in $\S 2$ and illustrate its many uses in $\S 3$. We discuss our results in $\S 4$.

\section{THE CONSTRUCTION OF THE SDSS STRIPE 82 STANDARD STAR CATALOG}

\subsection{Overview of SDSS Imaging Data}

SDSS uses a dedicated $2.5 \mathrm{~m}$ telescope (Gunn et al. 2006) to provide homogeneous and deep $(r<22.5)$ photometry in five bandpasses (Fukugita et al. 1996; Gunn et al. 1998, 2006; Smith et al. 2002; Hogg et al. 2001) repeatable to $0.02 \mathrm{mag}$ ( $\mathrm{rms}$ for sources not limited by photon statistics; Ivezić et al. 2003) and with a zero-point uncertainty of $\sim 0.02-0.03$ (Ivezić et al. 2004a). The survey sky coverage of close to $10,000 \mathrm{deg}^{2}$ in the northern Galactic cap and $\sim 300 \mathrm{deg}^{2}$ in the southern Galactic hemisphere will result in photometric measurements for well over 100 million stars and a similar number of galaxies. ${ }^{20}$ Astrometric positions are accurate to better than $0.1^{\prime \prime}$ per coordinate ( $\mathrm{rms}$ ) for sources with $r<20.5 \mathrm{mag}$ (Pier et al. 2003), and the morphological informa-

\footnotetext{
${ }^{19}$ Available at http://www.lsst.org/Science/lsst_baseline.shtml.

20 The recent Data Release 5 (J. K. Adelman-McCarthy et al. 2007, in preparation) lists photometric data for 215 million unique objects observed in $8000 \mathrm{deg}^{2}$ of sky; see http://www.sdss.org/dr5/.
}

tion from the images allows reliable star-galaxy separation to $r \sim$ 21.5 mag (Lupton et al. 2002; Scranton et al. 2002).

Data from the imaging camera (30 photometric, 12 astrometric, and 2 focus CCDs; Gunn et al. 1998) are collected in driftscan mode. The images that correspond to the same sky location in each of the five photometric bandpasses (these five images are collected over $\sim 5$ minutes, with $54 \mathrm{~s}$ for each exposure) are grouped together for simultaneous processing as a field. A field is defined as a $36 \mathrm{~s}$ (1361 pixels, or 9'; see Stoughton et al. 2002) long and 2048 pixels wide $\left(13^{\prime}\right)$ stretch of drift-scanning data from a single column of CCDs (sometimes called a "scan line"; for more details, see Stoughton et al. 2002; Abazajian et al. 2003, 2004, 2005; Adelman-McCarthy et al. 2006). Each of the six scan lines (together called a "strip") is $13^{\prime}$ wide. The 12 interleaved scan lines (or two strips) are called a "stripe" ( $\sim 2.5^{\circ}$ wide).

\subsection{The Photometric Calibration of SDSS Imaging Data}

SDSS $2.5 \mathrm{~m}$ imaging data are photometrically calibrated using a network of calibration stars obtained in $152041.5 \times 41.5 \mathrm{arcmin}^{2}$ transfer fields, called "secondary patches." These patches are positioned throughout the survey area and are calibrated using a primary standard star network of 158 stars distributed around the northern sky (so-called USNO standards; Smith et al. 2002). The primary standard star network is tied to an absolute flux system by the single F0 subdwarf star BD +17 4708, whose absolute fluxes in the SDSS filters are taken from Fukugita et al. (1996). The secondary patches are grouped into sets of four and are observed by the Photometric Telescope (PT; Tucker et al. 2006) in parallel with observations of the primary standards. A set of four patches spans all 12 scan lines of a survey stripe along the width of the stripe, and the sets are spaced along the length of a stripe at roughly $15^{\circ}$ intervals, which corresponds to $1 \mathrm{hr}$ of scanning at the sidereal rate.

SDSS $2.5 \mathrm{~m}$ magnitudes are reported on the "natural system" of the $2.5 \mathrm{~m}$ telescope defined by the photon-weighted effective wavelengths of each combination of SDSS filter, CCD response, telescope transmission, and atmospheric transmission at a reference air mass of 1.3 as measured at APO. ${ }^{21}$ The magnitudes are referred to as the ugriz system (which differs from the "primed" system, $u^{\prime} g^{\prime} r^{\prime} i^{\prime} z^{\prime}$, that is defined by the USNO standards). ${ }^{22}$ The reported magnitudes ${ }^{23}$ are corrected for the atmospheric extinction (using simultaneous observations of standard stars by the PT) and thus correspond to measurements at the top of the atmosphere 24 (except for the fact that the atmosphere has an impact on the wavelength dependence of the photometric system response). The magnitudes are reported on the AB system (Oke \& Gunn 1983), defined such that an object with a specific flux of $F_{\nu}=3631 \mathrm{Jy}$ has $m=0$ (i.e., an object with $F_{\nu}=$ const. has an AB magnitude equal to the Johnson $V$ magnitude at all wavelengths). In summary, given a specific flux of an object at the top of the atmosphere, $F_{\nu}(\lambda)$, the reported SDSS $2.5 \mathrm{~m}$ magnitude in a given

\footnotetext{
21 Transmission curves for the SDSS $2.5 \mathrm{~m}$ photometric system are available at http://www.sdss.org/dr5/instruments/imager

${ }^{22}$ For the subtle effects that led to this distinction, see Stoughton et al. (2002), Smith et al. (2002), and http://www.sdss.org/dr5/algorithms/fluxcal.html.

23 SDSS uses a modified magnitude system (Lupton et al. 1999), which is virtually identical to the standard astronomical Pogson magnitude system at high signal-to-noise ratios relevant here.

${ }^{24}$ The same atmospheric extinction correction is applied irrespective of the source color; within the relevant air-mass range $(X<1.5)$, the systematic errors this introduces are less than $1 \%$ for stars with effective temperatures in the $3000-$ $20,000 \mathrm{~K}$ range. For objects with significantly different spectral energy distributions, such as quasars and supernovae, the errors may be several times larger. See also Maíz Apellániz (2006).
} 
band, $b=(u, g, r, i, z)$, corresponds to (modulo random and systematic errors, which are discussed later)

$$
m=-2.5 \log _{10}\left(\frac{F_{b}}{3631 \mathrm{Jy}}\right)
$$

where

$$
F_{b}=\int F_{\nu}(\lambda) \phi_{b}(\lambda) d \lambda .
$$

Here $\phi_{b}(\lambda)$ is the normalized system response ${ }^{25}$ for the given band,

$$
\phi_{b}(\lambda)=\frac{\lambda^{-1} S_{b}(\lambda)}{\int \lambda^{-1} S_{b}(\lambda) d \lambda},
$$

with the overall atmosphere plus system throughput, $S_{b}(\lambda)$, available from the Web site given in footnote $21\left[\phi_{b}(\lambda)\right.$ for the SDSS system is shown in Fig. 6, discussed below; see also $\S$ 2.5.2]. We reiterate that the normalization of reported magnitudes corresponds to a source at the top of the atmosphere, while the throughput $\phi_{b}(\lambda)$ includes the transmission of a standard atmosphere at a fiducial air mass of 1.3. Note also that it is only the shape of $S_{b}(\lambda)$, and not its overall normalization, that needs to be known to compute expected SDSS magnitudes of a source with a given $F_{\nu}(\lambda)$. That is, the SDSS photometric system is fully defined by the five scaleless functions $\phi_{b}(\lambda)$ (by definition, $\int \phi_{b} d \lambda=1$; see eq. [3]). In reality, for each ugriz band there are six devices in the SDSS camera (Gunn et al. 1998) whose $\phi_{b}$ values are slightly different (see $\S 2.5 .2$ ).

The quality of SDSS photometry stands out among the available large-area optical sky surveys (Ivezić et al. 2003, 2004a; Sesar et al. 2006). Nevertheless, the achieved accuracy is occasionally worse than the nominal $0.02-0.03 \mathrm{mag}$ ( $\mathrm{rms}$ scatter for sources not limited by photon statistics). Typical causes of substandard photometry include an incorrectly modeled point-spread function (PSF; usually due to fast variations of atmospheric seeing or the lack of a sufficient number of the isolated bright stars needed for modeling the PSF), unrecognized changes in atmospheric transparency, errors in photometric zero-point calibration, effects of crowded fields at low Galactic latitudes, an undersampled PSF in excellent seeing conditions $\left(\lesssim 0.8^{\prime \prime}\right.$; the pixel size is $0.4^{\prime \prime}$ ), incorrect flat-field or bias vectors, and scattered light erroneously included in the flat field. Such effects can conspire to increase the photometric errors to levels as high as 0.05 mag (with a frequency, at that error level, of roughly one field per thousand). However, when multiple scans of the same sky region are available, many of these errors can be minimized by properly averaging the photometric measurements.

\subsection{The Choice of Cataloged Magnitudes}

The SDSS photometric pipeline ( photo; Lupton et al. 2002) measures several types of magnitudes, including aperture, PSF, and model magnitudes. Here we briefly describe each type of magnitude (for more details, see Stoughton et al. 2002 and the SDSS Web site $)^{26}$ and justify the choice of PSF magnitudes for catalog construction.

\footnotetext{
${ }^{25}$ The term $\lambda^{-1}$ in eq. (3) reflects the fact that CCDs are photon-counting devices; for more details, see Appendix A in Maíz Apellániz (2006).

${ }^{26}$ Available at http://www.sdss.org.
}

\subsubsection{Aperture Magnitudes}

Aperture magnitudes computed by photo are based on the flux contained within the aperture with a radius of $7.43^{\prime \prime}$. While an aperture magnitude is the most robust flux estimate for unsaturated sources at the bright end (because it is essentially seeingindependent), these magnitudes do not have good noise properties at the faint end where sky noise dominates (e.g., for a given maximum photometric error, PSF magnitudes reach 1-1.5 mag fainter than aperture magnitudes). In order to improve the depth of the standard star catalog, we opt not to use aperture magnitudes, except for quality tests at the bright end.

\subsubsection{Point-Spread Function Magnitudes}

The PSF flux is computed using the PSF as a weighting function. While this flux is optimal for faint point sources (in particular, it is vastly superior to aperture photometry at the faint end), it is also sensitive to inaccurate PSF modeling as a function of position and time. Even in the absence of atmospheric variations, the SDSS telescope and camera optics deliver images whose FWHMs vary by up to $15 \%$ from one side of a CCD to the other; the worst effects are seen in the chips farthest from the optical axis. Moreover, since the atmospheric seeing varies with time, the delivered image quality is a complex two-dimensional function even on the scale of a single frame. Without accounting for this spatial variation, the PSF photometry would have errors up to $0.10-$ 0.15 mag. The description of the PSF is also critical for star-galaxy separation and for unbiased measures of the shapes of nonstellar objects.

The SDSS imaging PSF is modeled heuristically in each band and each camera column using a Karhunen-Loéve(KL) transform (Lupton et al. 2002). Using stars brighter than roughly 20 mag, the PSF from a series of five frames is expanded into eigenimages, and the first three terms are retained. The variation of these coefficients is then fit up to a second-order polynomial in each chip coordinate. The failure of this KL expansion, typically due to an insufficient number of stars or an exceedingly complex PSF, results in occasional problems with PSF photometry. The main failure mode is inaccurate determination of aperture corrections which statistically tie PSF magnitudes to aperture magnitudes using bright stars.

\subsubsection{Model Magnitudes}

Just as the PSF magnitudes are optimal measures of the fluxes of stars, the optimal measure of the flux of a galaxy uses a matched galaxy model. With this in mind, the photometric pipeline fits two models to the two-dimensional image of each object in each band: a pure de Vaucouleurs profile and a pure exponential profile. ${ }^{27} \mathrm{Be}-$ cause the models are convolved with a double-Gaussian fit to the PSF, the seeing effects are accounted for. Aperture corrections are applied to make these model magnitudes equal the PSF magnitudes in the case of an unresolved object.

\subsubsection{The Choice of Magnitudes for the Standard Star Catalog}

A comparison between aperture, PSF, and model magnitudes for unresolved sources is done automatically for every SDSS observing run (runQA pipeline; Ivezić et al. 2004a). An analysis of over 200 runs indicates that model magnitudes are more robust than PSF magnitudes: PSF magnitudes show systematic offsets of 0.05 mag from aperture magnitudes 3 times more often than model magnitudes (roughly once per thousand fields), as model

\footnotetext{
${ }^{27}$ For more details, see http://www.sdss.org/dr5/algorithms/photometry.html.
} 
fits have more degrees of freedom. On the other hand, an analysis of repeated scans indicates that estimates of photometric errors by the photometric pipeline are more accurate for PSF magnitudes (agreeing at the 10\% level with the measured values; see Ivezić et al. 2003; Scranton et al. 2005) than for model magnitudes (which are smaller than the measured values by, typically, 30\%-50\%). Because the rejection of likely variable sources, which relies on accurate photometric error estimates, is an important step in the construction of the standard star catalog (see below), we choose to use PSF magnitudes to construct the catalog.

\subsection{Catalog Construction}

Using 58 SDSS-I runs from stripe 82 (approximately $20^{\mathrm{h}}<$ $\alpha_{\mathrm{J} 2000.0}<4^{\mathrm{h}}$ and $\left|\delta_{\mathrm{J} 2000.0}\right|<1.266^{\circ}$, but not all runs extend over the entire right ascension range) obtained in mostly photometric conditions (as indicated by the PT calibration residuals, infrared cloud camera, ${ }^{28}$ and tests performed by the runQA pipeline), candidate standard stars from each run are selected by requiring the following:

1. That objects are classified as STAR (based on the difference between model and PSF magnitudes); this morphological classification really means unresolved (point) sources (e.g., quasars are also included).

2. That they have quoted photometric errors in the PSF magnitude (as computed by the photometric pipeline) smaller than $0.05 \mathrm{mag}$ in at least one band.

3. That the processing flags BRIGHT, SATUR, BLENDED, or EDGE are not set in any band. ${ }^{29}$

These criteria select unsaturated point sources with sufficiently high signal-to-noise ratio per single observation to approach final photometric errors of 0.02 mag or smaller.

After matching all detections of a single source across runs (using a $1^{\prime \prime}$ matching radius), various photometric statistics such as unweighted mean, median, and their standard errors; rms scatter; number of observations; and $\chi^{2}$ per degree of freedom are computed for magnitudes in each band. We use errors reported by the photometric pipeline to compute $\chi^{2}$ and note that systematic errors common to all runs do not contribute to its value. This initial catalog of multiepoch observations includes 1.4 million point sources with at least four observations in each of the $g, r$, and $i$ bands. The median number of observations per source and band is 10 , and the total number of photometric measurements is $\sim 57$ million.

The distributions of the median magnitudes, their standard errors, $\chi^{2}$, and the number of observations for a subset of these sources are shown in Figure 1. The random errors in the median magnitude [computed as $0.928^{*} \mathrm{IQR} /(N-1)^{1 / 2}$, where IQR is the $25 \%-75 \%$ interquartile range of the individual measurement distribution and $N$ is the number of measurements; note that the error of the median for a Gaussian distribution is $25 \%$ larger than the error of the mean; Lupton 1993] are below 0.01 mag at the bright end. These errors are reliably computed by the photometric pipeline, as indicated by the $\chi^{2}$ distributions. The distributions of these sources in color-magnitude and color-color diagrams, constructed using median magnitudes, are shown in Figure 2. For a detailed interpretation of these diagrams, see Lenz et al. (1998), Fan (1999), Finlator et al. (2000), Helmi et al. (2003),

\footnotetext{
${ }^{28}$ For more details about the camera, see http://www.apo.nmsu.edu/Telescopes/ SDSS/eng.papers/19910801/19910801.html and Hogg et al. (2001).

${ }^{29}$ For more details about processing flags, see http://www.sdss.org/dr5/ products/catalogs/flags.html and Stoughton et al. (2002).
}

and Ivezić et al. (2006). It is evident that the sample is dominated by stars.

\subsubsection{Selection of Candidate Standard Stars}

Adopted candidate standard stars must have at least four observations and, to avoid variable sources, $\chi^{2}$ less than 3 in the $g r i$ bands (the same requirements are later applied in the $u$ and $z$ bands when using the catalog for calibration, as we discuss further below). We also limit the right ascension to the range from $20^{\mathrm{h}} 34^{\mathrm{m}}$ to $4^{\mathrm{h}} 00^{\mathrm{m}}$, which provides a simple areal definition (together with $\left|\delta_{\text {J2000.0 }}\right|<1.266^{\circ}$ ) of a $282 \mathrm{deg}^{2}$ large rectangular region, while excluding only a negligible fraction of stars. With the final condition that the standard error for the mean magnitude in the $r$ band is smaller than $0.05 \mathrm{mag}$, these requirements result in a catalog with slightly over 1 million sources $(1,006,849)$ with 42 million photometric measurements. Of those, 563,908 have a random error for the median magnitude in the $r$ band smaller than $0.01 \mathrm{mag}$, and for 405,954 stars this is true in all three of the gri bands. Subsets of 92,905 and 290,299 stars satisfy these requirements in the ugri and griz bands, and 91,853 stars satisfy this in all five bands. The distributions of candidate standard stars that satisfy the above selection criteria in all five bands in color-magnitude and colorcolor diagrams are shown in Figure 3.

For comparison, the distribution of sources that were rejected as variable ( $\chi^{2}$ greater than 3 in at least one of the gri bands) in color-magnitude and color-color diagrams is shown in Figure 4. As is evident from a comparison with Figure 3, the distribution of variable sources in the color-color diagrams is markedly different from that of nonvariable sources. It is especially striking how low-redshift $(z<2.2)$ quasars are easily detected by their variability (for more details, see Ivezić et al. 2004b). However, it is fairly certain that not all variable sources are recognized as such because of the limited number of repeated observations $(\sim 10)$. For example, an eclipsing binary with a much shorter eclipse duration than the orbital period could easily escape detection. Analysis of the variable subsample is presented in a companion paper (B. Sesar et al. 2007, in preparation).

The sky density of all the sources and those selected as nonvariable are shown in Figure 5. At high Galactic latitudes $(|b| \sim$ $60^{\circ}$ ) the fraction of candidate standard star sources is $\sim 80 \%$.

\subsection{Systematic Photometric Errors}

Photometric errors computed by the photometric pipeline provide a good estimate of random errors in SDSS photometry, as demonstrated by the $\chi^{2}$ distributions shown in Figure 1. However, the measurements are also subject to systematic errors such as spatial dependence of the internal zero points (calibration errors) and the overall deviations of the internal SDSS zero points from an $\mathrm{AB}$ magnitude scale. Formally, the true $\mathrm{AB}$ magnitude of an object (defined by eq. [1]) in a given band, $m_{\text {true }}$, can be expressed as

$$
\left.m_{\text {true }}=m_{\text {cat }}+\delta_{m} \text { (R.A., decl. }\right)+\Delta_{m},
$$

where $m_{\text {cat }}$ is the cataloged magnitude, $\delta_{m}$ (R.A., decl.) describes the spatial variation of the internal zero-point error around $\Delta_{m}$ (thus, the average of $\delta_{m}$ over the cataloged area is zero by construction), and $\Delta_{m}$ is the overall (spatially independent) deviation of the internal SDSS system from a perfect $\mathrm{AB}$ system (the five values of $\Delta_{m}$ are equal for all the cataloged objects). Here we ignore systematic effects, e.g., device nonlinearity and bandpass variations between different camera columns, which depend on individual source properties such as brightness and color (but see $\S 2.5 .2$ below). 


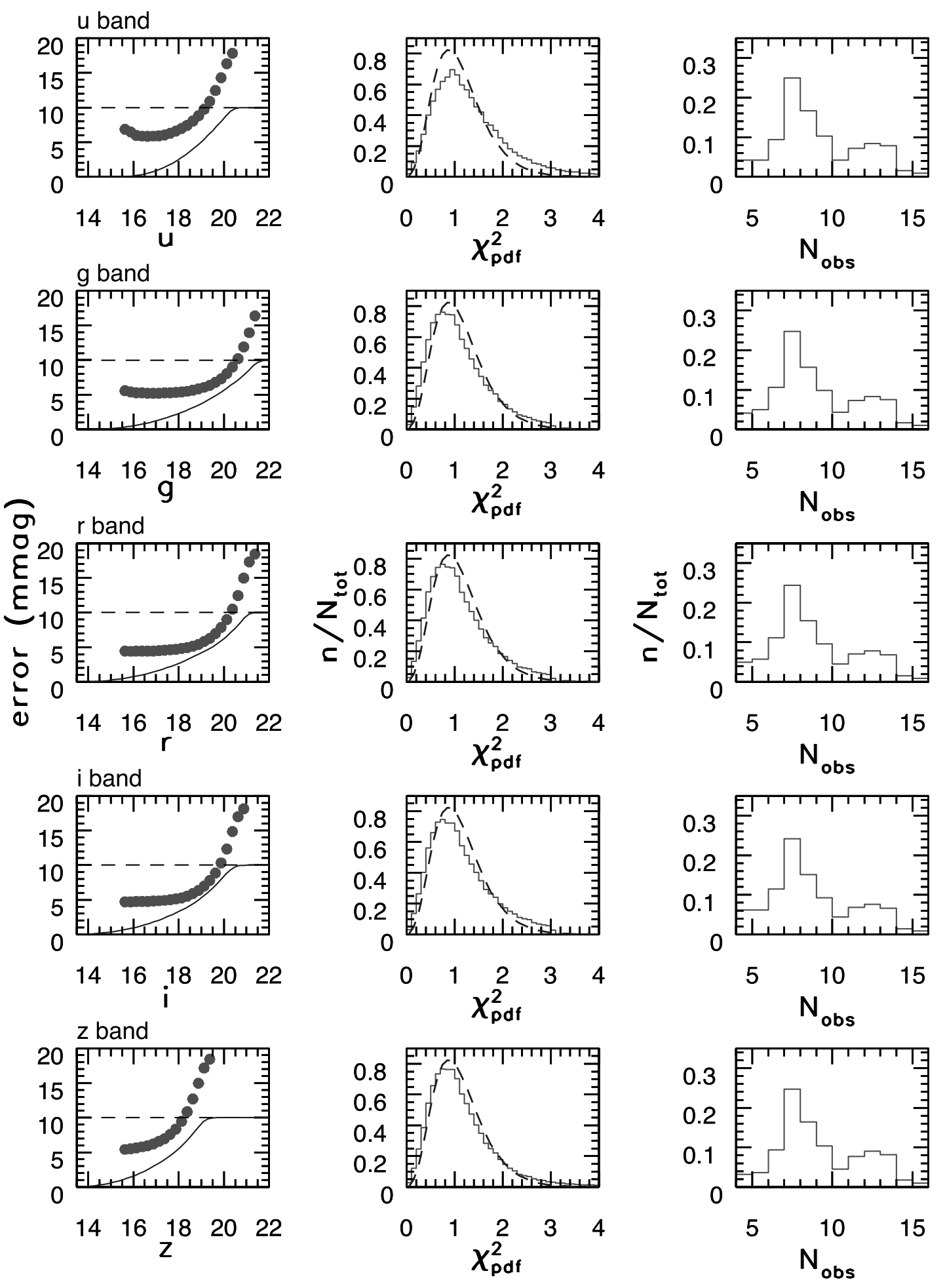

FIG. 1.-Median magnitude error as a function of magnitude (left column, symbols), the $\chi^{2}$ per degree of freedom distribution (middle column, solid line), and the number of observations in each band (right column) for candidate standard stars from SDSS stripe 82 (selected by $\chi^{2}<3$ in the gri bands). The solid lines in the left column show cumulative magnitude distribution normalized to 10 at the faint end. The dashed lines in the middle column show the $\chi^{2}$ per degree of freedom distribution for a Gaussian error distribution and 9 degrees of freedom. Its similarity with the measured distributions suggests that the magnitude errors computed by the photometric pipeline are reliable (they may be slightly underestimated, by about $10 \%$, in the $u$ band). [See the electronic edition of the Journal for a color version of this figure.]

The spatial variation of the internal zero-point error can be separated into "color" errors, relative to a fiducial band, say $r$, and an overall "gray" error (e.g., unrecognized temporal changes in atmospheric transparency due to gray clouds),

$$
\left.\delta_{m} \text { (R.A., decl. }\right)=\delta_{r} \text { (R.A., decl.) }+\delta_{m r} \text { (R.A., decl.). }
$$

Below, we discuss methods for estimating both the gray error $\delta_{r}$ (R.A., decl.) and the color errors $\delta_{m r}$ (R.A., decl.).

The deviation of the internal SDSS system from a perfect AB system, $\Delta_{m}$, can also be expressed relative to the fiducial $r$ band,

$$
\Delta_{m}=\Delta_{r}+\Delta_{m r}
$$



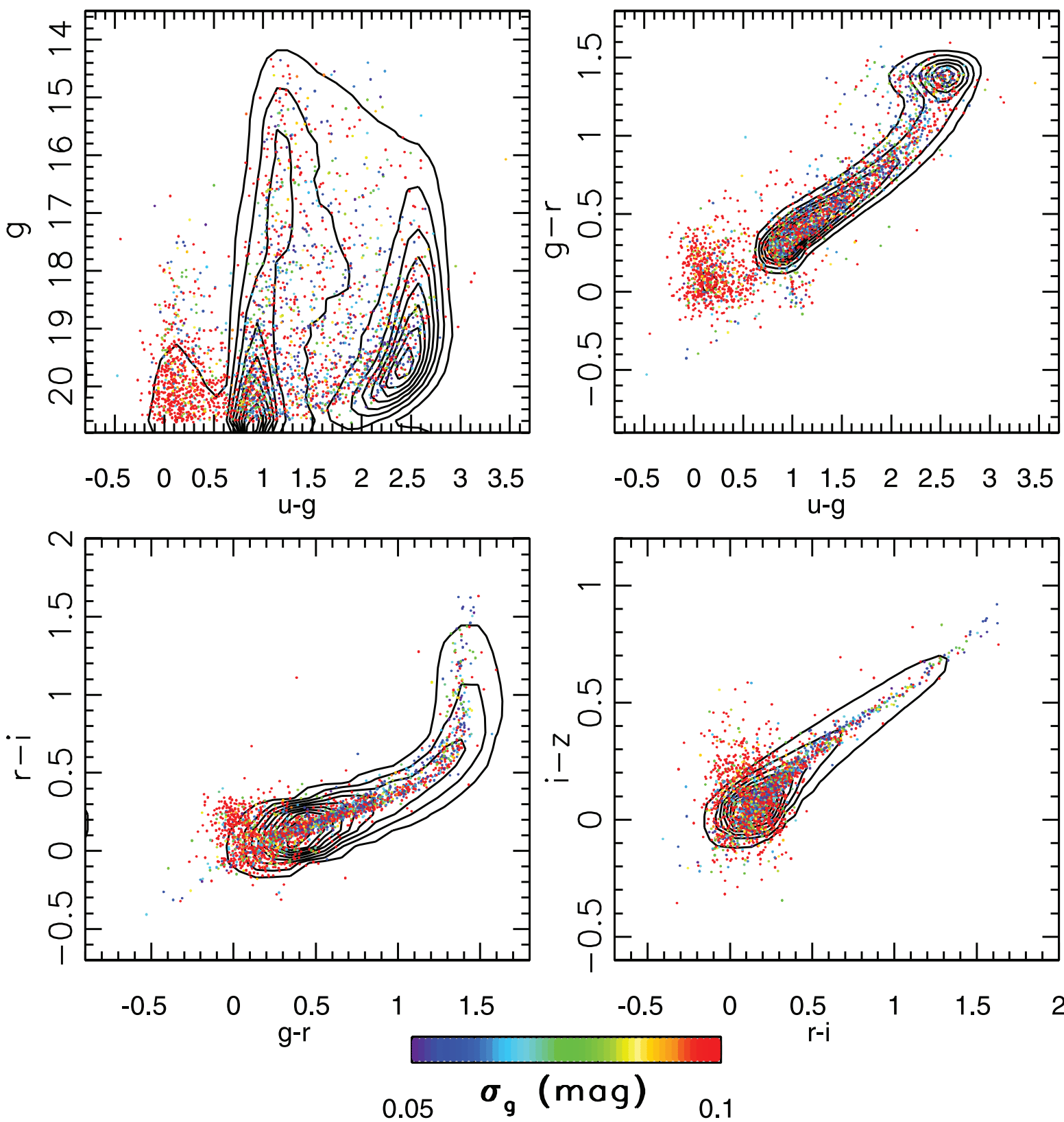

FIG. 2.-Comparison of the color-magnitude and color-color distributions for variable and nonvariable unresolved sources from SDSS stripe 82. The distributions of sources with an rms scatter of the $g$-band magnitude below $0.05 \mathrm{mag}$ are shown by linearly spaced contours (nonvariable sources). Sources with $320^{\circ}<$ R.A. $<330^{\circ}$, with rms scatter in the $g$ band larger than $0.05 \mathrm{mag}$ and $\chi^{2}$ greater than 3 , are shown by dots (variable sources). The dots are color-coded according to the observed rms scatter in the $g$ band (0.05-0.10 mag; see the legend, where red indicates variability larger than $0.1 \mathrm{mag})$. Note how low-redshift quasars $(u-g<0.6)$ and RR Lyrae stars $(u-g \sim 1.1, g-r \sim 0$; Ivezić et al. 2005) clearly stand out as variable sources (red dots).

The motivation for this separation is twofold. First, $\Delta_{m r}$ can be constrained by considering the colors (spectral energy distributions) of objects independently from the overall flux scale (this can be done using both external observations and models). Second, it is difficult to find a science result that crucially depends on knowing the "gray-scale" offset, $\Delta_{r}$, at the $1 \%-2 \%$ level. On the other hand, knowing the "band-to-band" offsets, $\Delta_{m r}$, with such an accuracy is important for many applications (e.g., photometric redshifts of galaxies, Type Ia supernova cosmology, and testing of stellar and galaxy models).

Fitting the SDSS spectra of hot white dwarfs to models, Eisenstein et al. (2006) determined the AB color corrections $\Delta_{m r}$ to be $-0.040,+0.000,+0.015$, and +0.030 mag for $m=u g i z$, respectively, with an uncertainty of $\sim 0.01-0.02 \mathrm{mag}$. Similar values of $\Delta_{m r}$, also based on hot white dwarfs, were obtained by Holberg \& Bergeron (2006): $-0.046,+0.001,+0.013$, and +0.024 mag for $m=u g i z$, respectively. It may be possible to determine these corrections with an uncertainty of $\sim 0.01 \mathrm{mag}$, and such efforts are in progress (J. Marriner 2007, private communication).

The overall gray flux scale calibration error, $\Delta_{r}$, is determined by the accuracy of the absolute flux calibration of fundamental standard BD +17 4708 (Fukugita et al. 1996), the accuracy of tying the primary standard star network to BD +174708 , the accuracy of transferring the primary standard star network to the secondary standard star network, and the accuracy of the calibration of the survey imaging data using the secondary standard star network. Given these numerous sources of error, it seems 

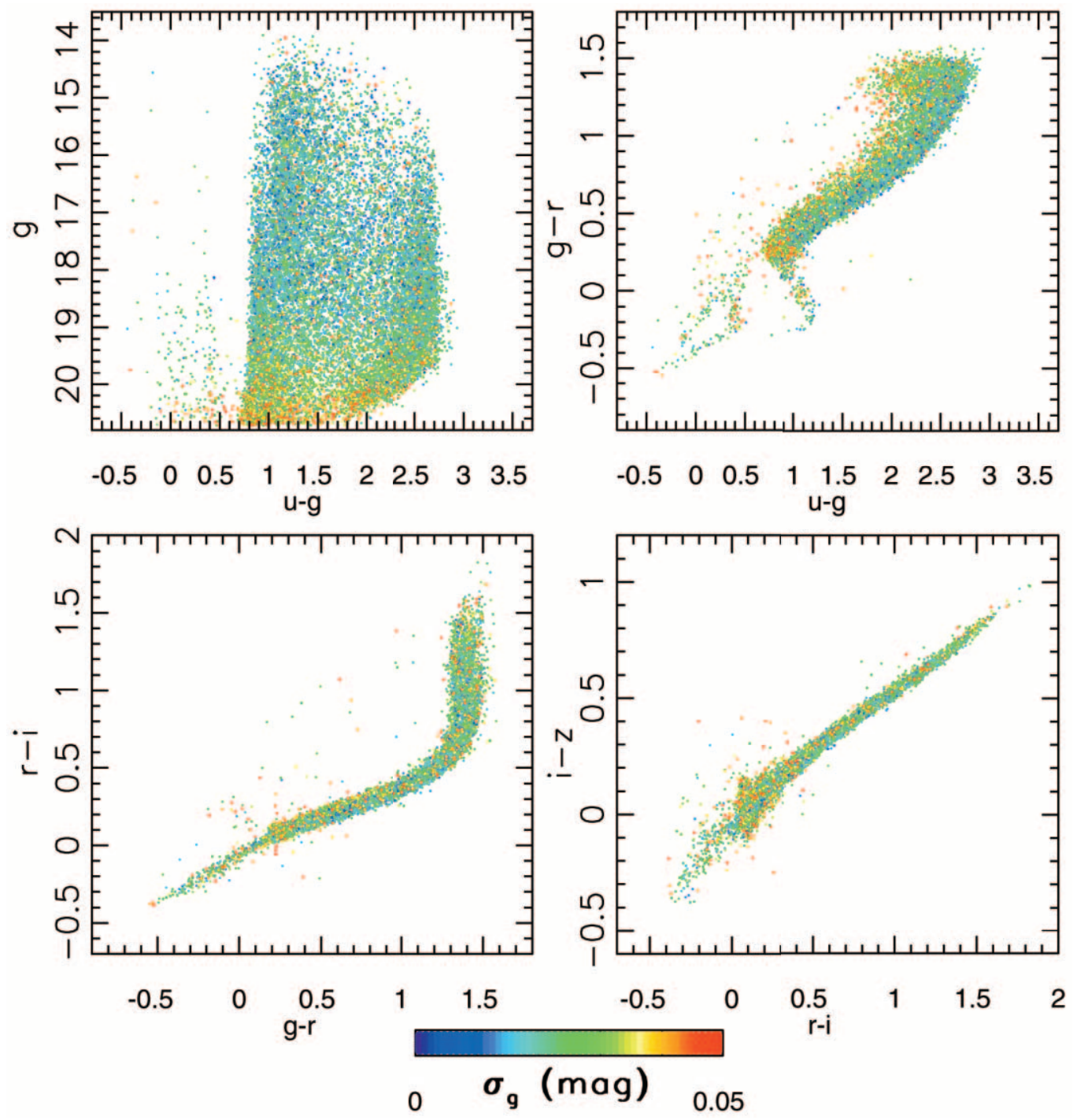

FIG. 3.-Color-magnitude and color-color distributions of candidate standard stars brighter than $g=20$. The dots are color-coded according to the observed rms scatter in the $g$ band ( $0-0.05$ mag; see the legend). Note the absence of quasars and RR Lyrae stars visible in Fig. 2.

unlikely that $\Delta_{r}<0.02 \mathrm{mag}$. On the other hand, formal analysis of all the error contributions, as well as a direct comparison to Hubble Space Telescope (HST) observations of hot white dwarfs (Eisenstein et al. 2006), suggests that $\Delta_{r}$ does not exceed $0.05 \mathrm{mag}$; in addition, the analysis of HST spectrophotometry and other ground-based data by Holberg \& Bergeron (2006) yields $\Delta_{r}=0.003 \mathrm{mag}$. Note, however, that all these uncertainties in the definition and transfer of the standard star network become moot if one accepts the following:

1. We do not need to know $\Delta_{r}$ exquisitely well for most scientific applications. ${ }^{30}$ Even if we do, this is just $a$ single number that modifies the cataloged photometry for all the sources and all the bands in the same fashion.

2. Uncertainties in the determination of $\Delta_{m r}$ are of the order of 0.01 mag.

3. We can constrain or correct for $\delta_{m}$ (R.A., decl.) at the 0.01 mag level.

\footnotetext{
${ }^{30}$ Here we assume that color offsets between different surveys and photometric systems can be treated in a fashion similar to that of the color offsets between SDSS bands. For example, hot white dwarfs could be used to compute the AB color correction, $\Delta_{m r}$, between the SDSS $z$ band and 2MASS $J$ band with an accuracy of $\sim 0.01-0.02 \mathrm{mag}$.
}

In other words, the band-to-band calibration can be fixed by adopting $\Delta_{m r}$, determining and correcting for $\delta_{m}$ (R.A., decl.) guarantees internal consistency, and the only remaining relatively free parameter is $\Delta_{r}$. Such a system is then no longer defined by a set of celestial standards but rather by the functions $\phi_{b}$. The catalog presented here is one realization of such a photometric system.

\subsubsection{Determination of $\delta_{m}$ (R.A., decl.)}

We now proceed to describe methods for constraining $\delta_{m}$ (R.A., decl.). The region covered by SDSS stripe 82 is an elongated rectangle with an aspect ratio of 1:50 and with the long side parallel to the celestial equator. Because of this large aspect ratio, and because different effects contribute to the right ascension and declination dependences of $\delta_{m}$, we assume that it can be expressed as a sum of independent functions of right ascension or declination, respectively,

$$
\delta_{m}(\text { R.A., decl. })=\delta_{m}^{\mathrm{ff}}(\text { decl. })+\delta_{m}^{\mathrm{ext}}(\text { R.A. }),
$$

with $\left\langle\delta_{m}^{\mathrm{ff}}(\text { decl. })\right\rangle_{\text {R.A. }}=0$ and $\left\langle\delta_{m}^{\text {ext }}\right.$ (R.A. $\left.)\right\rangle_{\text {decl. }}=0$, where $\langle\delta\rangle_{x}$ denotes the average of $\delta$ over direction $x$.

The first term, $\delta_{m}^{\mathrm{ff}}(\mathrm{decl}$.), is dominated by the errors in the flat-field vectors (for drift scanning, flat-field corrections are 

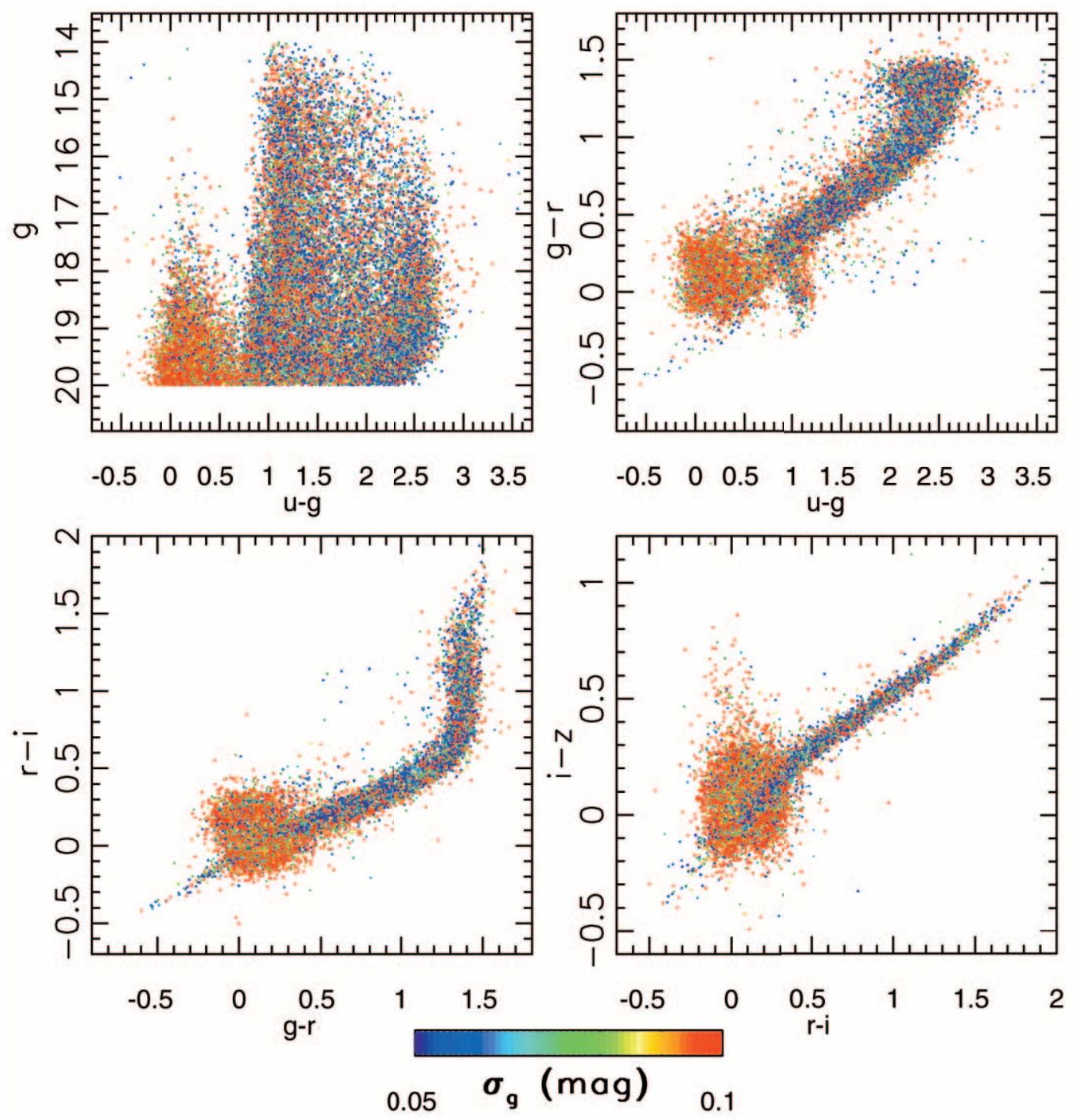

FIG. 4.- Same as Fig. 3, but only for variable sources and with a different color coding ( $0.05-0.10$ mag range of the $g$-band rms, instead of $0-0.05$ mag).

one-dimensional). The flat-field determination for SDSS was difficult due to scattered light, ${ }^{31}$ and there are probably systematic errors in the stellar photometry at the $0.01 \mathrm{mag}$ level in the griz bands and the 0.02 mag level in the $u$ band (perhaps somewhat larger at the edge of the imaging camera), as we demonstrate below. Since these systematic errors do not cancel when averaging many observing runs ( because most stars are always observed by the same CCD and fall on roughly the same position within the $\mathrm{CCD}), \delta_{m}^{\mathrm{ff}}$ (decl.) could be as large as $\sim 0.01-0.02$ mag on spatial scales much smaller than the chip width of $13^{\prime}$.

The second term, $\delta_{m r}^{\operatorname{ext}}$ (R.A.), is dominated by unrecognized fast variations of atmospheric extinction (e.g., due to cirrus), because for each observing run only a single zero point per CCD is determined. ${ }^{32}$ While such variations are uncorrelated for different runs, it is possible that they do not average out fully at the $1 \%$ level.

\footnotetext{
${ }^{31}$ For details, see http://www.sdss.org/dr5/algorithms/flatfield.html and Stoughton et al. (2002).

${ }_{32}$ Although not relevant for the equatorial runs discussed here, the slow change of atmospheric extinction due to varying air mass is accounted for; the response of the telescope and CCDs is stable at the $<1 \%$ level on single-night timescales.
}

\subsubsection{The Six ugriz Photometric Systems}

Before we describe methods for determining $\delta_{m}$ (R.A., decl.), we address the bandpass differences between the six camera columns. The bandpasses had been measured for each CCD using a monochromator (M. Doi et al. 2007, in preparation) and found not to be identical, as shown in Figure 6 . These differences between the bandpasses induce color-term errors in the reported SDSS photometry because the magnitudes of calibration stars obtained by the PT are transformed to the $2.5 \mathrm{~m}$ system using a single set of color terms. ${ }^{33}$ In other words, the color difference between a blue star and a red star depends on which camera column the stars fall on; at the $\sim 1 \%$ accuracy level discussed here, there are six SDSS ugriz systems.

We used the measured response curves to generate synthetic ugriz photometry corresponding to six camera columns (via eq. [1]) for 175 stars from the atlas of Gunn \& Stryker (1983). The differences between the predicted magnitudes for each camera column and the values generated with the response curves which define the SDSS system ${ }^{34}$ represent photometric corrections due

\footnotetext{
${ }^{33}$ See http://www.sdss.org/dr5/algorithms/jeg_photometric_eq_dr1.html.

${ }^{34}$ Transmission curves for the SDSS $2.5 \mathrm{~m}$ photometric system are available at http://www.sdss.org/dr5/instruments/imager.
} 


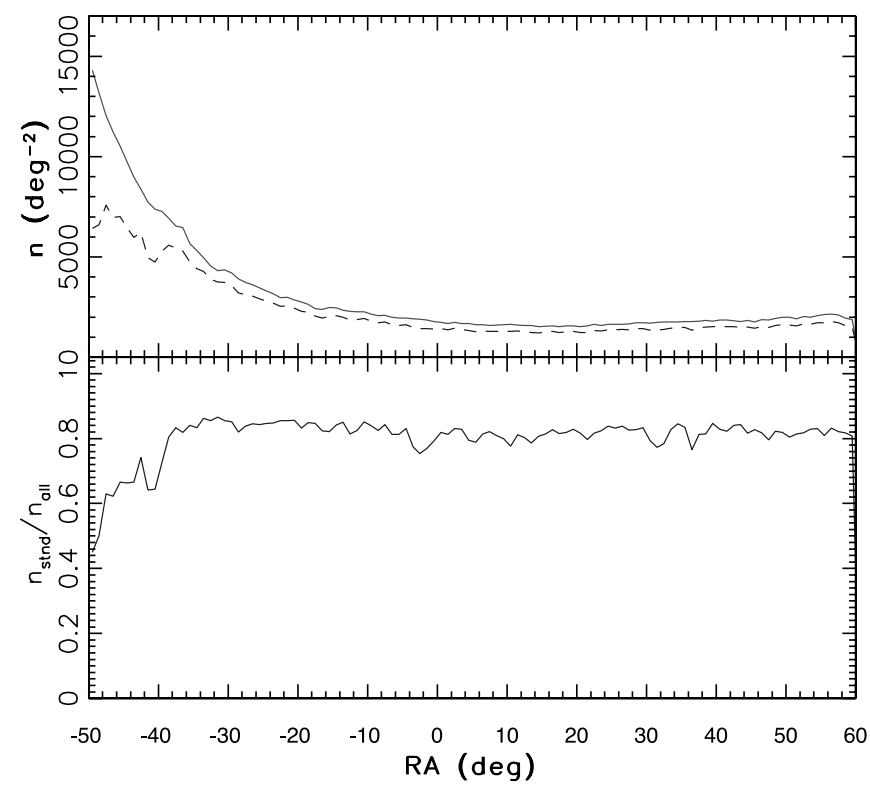

FIG. 5.-Top: Sky density of all the point sources with at least four observations in each of the $g, r$, and $i$ bands (solid line) and of selected candidate standard (nonvariable) stars (dashed line). Bottom: Ratio of the two curves shown in the top panel. For reference, Galactic coordinates $(l, b)$ are $(46,-24),(96,-60)$, and $(190,-37)$ for $\alpha_{\mathrm{J} 2000.0}=-50^{\circ}, 0^{\circ}$, and $60^{\circ}$ (at $\left.\delta_{\mathrm{J} 2000.0}=0^{\circ}\right)$. [See the electronic edition of the Journal for a color version of this figure.]

to bandpass differences. These corrections may be as large as 0.02 mag for the reddest stars but fortunately admit simple linear fits as a function of color ( $u-g$ for the $u$ band, $g-r$ for $g$ and $r$, and $r-i$ for $i$ and $z$ ). The $u$ band is the only one where piecewise fits are required (in the range $0.7<u-g<1.3$, the $g-r$ color provides a better fit than the $u-g$ color). We have applied these best-fit corrections to all sources in the catalog. The median and rms scatter for the distributions of corrections evaluated for all stars, and for each color and camera column combination, are listed in Table 1.

The bandpass differences have the largest impact on the $i-z$ color of red sources. The color-term errors result in a rotation of the stellar locus in the $i-z$ versus $r-i$ color-color diagram (see Fig. 2, bottom right), and we use this fact to demonstrate the improvement in photometry due to applied corrections. We use the mean position of the stellar locus to "predict" the $i-z$ color from the measured $r-i$ color and compute the difference between predicted and measured $i-z$ colors separately for blue $(0.1<r-$ $i<0.2)$ and red $(0.8<r-i<1.4)$ stars and in small bins of declination (cross-scan direction). The difference of these residuals (blue vs. red stars) effectively measures the locus position angle and is not sensitive to photometric zero-point errors and flat-field errors (which can only induce locus shifts that have no effect on this test because they cancel out).

The top panel in Figure 7 shows the median $i-z$ residuals before applying corrections for different transmission curves, and the bottom panel shows the results based on corrected photometry. The rms scatter decreases from 9 to 3 mmag after applying the corrections. The remaining deviations of residuals from zero could be due to the fact that we have not corrected for the dependence of the flat fields on source color (measurements of this dependence are not available). Because this is currently the only available SDSS catalog with photometry corrected for bandpass differences, it effectively represents a practical definition of the SDSS photometric system (i.e., it provides photometry for real sources on the sky; the system is formally defined by $\phi_{b}$, of course).
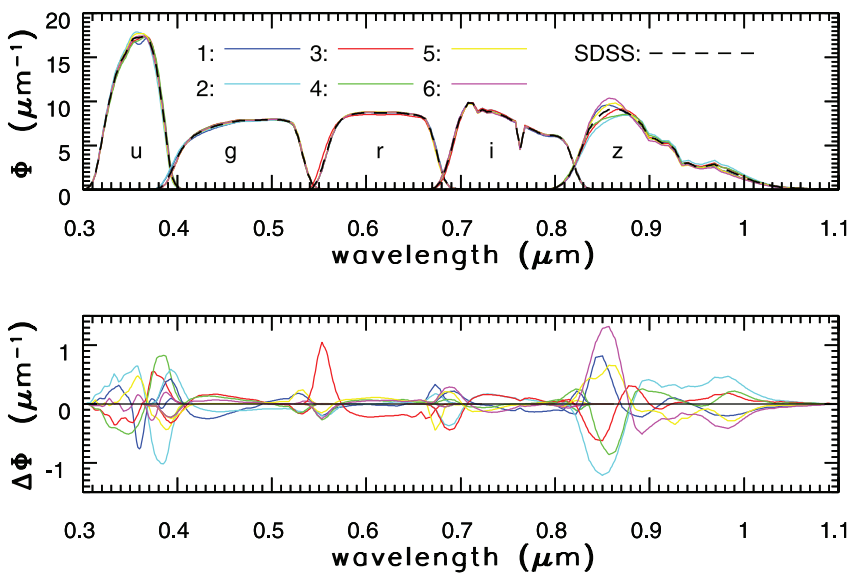

FIG. 6.-Top: Comparison of the five transmission curves, $\phi$ (see eq. [3] for definition), that define the SDSS photometric system (dashed lines) to measured transmission curves sorted by camera column and shown by solid lines, as marked in the panel (the area under each curve is unity by definition). Bottom: Differences between the measured transmission curves and the curves that define the system. These differences induce color terms that result in systematic photometric errors as a function of source color. The largest color terms are present for the $z$ band in camera columns 2 and 6 , with the errors well described by $\Delta z_{2}=z_{2}-z_{\text {SDSS }}=$ $-0.019(r-i)$ and $\Delta z_{6}=+0.017(r-i)$ mag, respectively.

\subsubsection{Other Sources of Systematic Errors}

The nonlinearity of the detectors (as a function of source brightness) has also been measured in situ by $\mathrm{M}$. Doi et al. and found to be at most a couple of percent effect over the relevant dynamic range. ${ }^{35}$ These corrections are determined with a sufficient accuracy ( $<5$ mmag impact on photometry) and are already implemented in the photometric pipeline (i.e., before performing photometric calibration).

Similarly to the camera column-to-column bandpass differences, variations in the wavelength dependence of atmospheric transmissivity can also induce systematic errors that depend on source colors. Assuming a standard atmosphere, using synthetic photometry for stars from the Gunn-Stryker atlas we find that this effect can induce offsets of up to $\sim 0.01 \mathrm{mag}$ for the $u-g$ and $g-r$ colors when air mass is varied by 0.3 from its fiducial value of 1.3. However, because all stripe 82 data are obtained at the same air mass, this effect is not relevant for the catalog discussed here. At least in principle, similar and potentially larger errors could be induced even at a constant air mass if the wavelength dependence of atmospheric transmissivity is significantly different from the assumed standard atmosphere. Given that such errors would probably average out, and that there are no available measurements of the wavelength dependence of atmospheric transmissivity ${ }^{36}$ for the SDSS data considered here, we ignore this effect hereafter.

\subsection{Determination of Flat-Field Corrections}

We use two methods based on SDSS data to constrain $\delta_{m}$ (R.A., decl.): a direct comparison with the secondary standard

\footnotetext{
${ }^{35}$ For details, see http://www.sdss.org/dr5/instruments/imager/nonlinearity .html.

${ }^{36}$ Some handle on the stability of the wavelength dependence of atmospheric transmissivity can be obtained by studying first-order extinction coefficients determined by the photometric calibration pipeline. They show a cyclic variation during the year, with an rms scatter of residuals around the mean relation of $0.01 \mathrm{mag}$. The amplitude of the yearly variation of the $r$-band extinction coefficient is $\sim 20 \%$ about the mean value, and the wavelength dependence of the variation appears consistent with the addition of a gray opacity source during the summer months.
} 
TABLE 1

The Color-Term Corrections

\begin{tabular}{|c|c|c|c|c|c|c|c|c|c|c|}
\hline Column & $\langle d u\rangle$ & $\sigma_{d u}$ & $\langle d g\rangle$ & $\sigma_{d g}$ & $\langle d r\rangle$ & $\sigma_{d r}$ & $\langle d i\rangle$ & $\sigma_{d i}$ & $\langle d z\rangle$ & $\sigma_{d z}$ \\
\hline 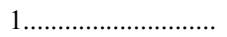 & 1.0 & 0.3 & 1.8 & 0.4 & -1.2 & 0.3 & 0.0 & 0.2 & 0.6 & 0.5 \\
\hline $2 \ldots \ldots \ldots \ldots \ldots \ldots \ldots \ldots \ldots$ & 11.0 & 2.3 & 7.6 & 1.6 & -1.1 & 0.2 & -0.0 & 0.2 & -0.8 & 1.3 \\
\hline З & -5.4 & 0.8 & -2.0 & 0.0 & 2.9 & 1.0 & -0.2 & 0.3 & -0.2 & 0.5 \\
\hline 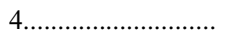 & -9.5 & 2.1 & -1.0 & 0.2 & -0.9 & 0.1 & 0.0 & 0.0 & -0.4 & 0.3 \\
\hline 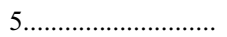 & 3.9 & 1.0 & -4.4 & 1.3 & -0.0 & 0.2 & -0.2 & 0.1 & 0.4 & 0.7 \\
\hline 6........................... & -0.5 & 0.8 & -2.6 & 0.4 & -0.6 & 0.1 & -0.2 & 0.3 & 0.9 & 1.2 \\
\hline
\end{tabular}

NotE.-The median and rms scatter for photometric corrections that place the measurements in six camera columns on the survey system (in millimagnitudes).

star network ( $\S 2.6 .2)$ and a method based on stellar colors $(\S 2.6 .1)$. Each of these methods has its advantages and disadvantages. We perform tests of the final catalog quality using a method based on the photometric redshift relation for galaxies $(\S 2.7 .2)$, and we also compare the SDSS photometry to an independent set of standards provided by Stetson $(2000,2005)$ in $\S 2.7 .3$.

A determination of the $\delta_{m}$ (R.A., decl.) error from a direct comparison with the secondary standard star network (hereafter "PT comparison") might be considered the best method a priori. However, it is quite possible that the secondary standard star network itself, from which this catalog is calibrated, may induce a spatial variation of the photometric zero points at the 0.01 mag level (Smith et al. 2002). In addition, there are not enough stars to constrain $\delta_{m}^{\mathrm{ff}}(\mathrm{decl}$.) with sufficient spatial resolution (say, at least $\sim 100$ pixels, or $\sim 0.01^{\circ}$ ). For example, there are $\sim 20,000 \mathrm{sec}-$ ondary standards from stripe 82 in the averaged catalog that are not saturated in the gri bands in the $2.5 \mathrm{~m}$ scans and have PT errors smaller than 0.03 mag $(\sim 8000$ stars are usable in the $z$ band and only $\sim 3000$ in the $u$ band). If these stars are binned in the declination direction every $0.01^{\circ}$ (250 bins, each 90 pixels wide), $\delta_{m}^{\mathrm{ff}}$ in each bin can be constrained to about $\sim 0.005 \mathrm{mag}(0.01 \mathrm{mag}$ in the $u$ band). This is barely sufficient in the gri bands and cannot
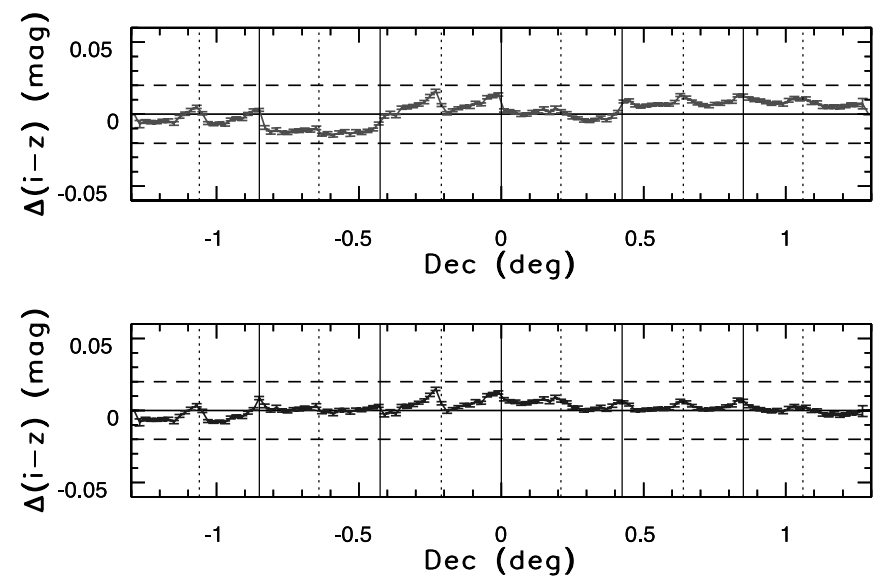

FIG. 7.- Test based on the stellar locus position angle in the $i-z$ vs. $r-i$ colorcolor diagrams that demonstrates the existence of color terms between different camera columns. The $y$-axis shows the difference in the $i-z$ color residuals of blue $(0.1<r-i<0.2)$ and red $(0.8<r-i<1.4)$ stars, with the residuals computed as the difference between measured $i-z$ colors and those predicted using the mean stellar locus (see Fig. 3, bottom right panel). The vertical solid lines mark the approximate boundaries between different camera columns, with the vertical dashed lines marking approximate boundaries between the "north" and "south" strips in a stripe (see $\S 2.1$ for definitions). The top panel shows the results before applying corrections for different transmission curves (see Fig. 6), and the bottom panel shows the results based on corrected photometry. It is evident that the residuals in the bottom panel are much smaller, with rms scatter decreasing from 9 to $3 \mathrm{mmag}$. [See the electronic edition of the Journal for a color version of this figure.] provide satisfactory constraints on the flat-fielding errors in the $u$ band (where, unfortunately, these errors are the largest). Similarly, $\delta_{m}^{\text {ext }}$ (R.A.) can be constrained in $0.5^{\circ}$ wide right ascension bins with a similar accuracy, but the secondary standard stars are not uniformly distributed in right ascension. For these reasons, we combine the PT comparison with the stellar locus method to determine flat-field corrections.

\subsubsection{Color Corrections from the Stellar Locus Method}

The stellar distribution in color-color space at high Galactic latitudes ${ }^{37}(|b|>30)$ is remarkably uniform at the faint flux levels probed by SDSS, as discussed in detail by Ivezić et al. (2004a). Systematic photometric errors, other than an overall gray error, manifest themselves as shifts in the position of the stellar locus that can be tracked using the four principal colors (swxy) defined by Ivezić et al. (2004a). These colors are linear combinations of magnitudes,

$$
P_{2}=A u+B g+C r+D i+E z+F,
$$

where $P_{2}=s, w, x, y$, and measure the distance from the center of the locus in various two-dimensional projections of the fourdimensional stellar color distributions ( $s$, perpendicular to the blue part of the locus in the $g-r$ vs. $u-g$ plane; $w$, perpendicular to the blue part in the $r-i$ vs. $g-r$ plane; $x$, perpendicular to the red part with $g-r \sim 1.4$ in the $r-i$ vs. $g-r$ plane; and $y$, perpendicular to the locus in the $i-z$ vs. $r-i$ plane). The matrix of coefficients $A-F$ is listed in Table 2 (for more details, see Ivezić et al. 2004a). Of course, the measurements must be corrected for the effects of interstellar dust extinction; we use maps provided by Schlegel et al. (1998, hereafter SFD98). The properties of two of these colors $(s$ and $w$ ) are illustrated in Figure 8.

The fact that the median principal colors are close to zero shows that the averaging procedure did not induce any shifts in zero points compared to the average of 291 SDSS runs which were used to define the principal colors (Ivezić et al. 2004a). The same

\footnotetext{
${ }^{37}$ At low Galactic latitudes several effects, discussed below, prevent the use of this method for calibration purposes.
}

TABLE 2

The swxy Principal Color Definitions

\begin{tabular}{|c|c|c|c|c|c|c|}
\hline PC & $A$ & $B$ & $C$ & $D$ & $E$ & $F$ \\
\hline$s+\ldots \ldots \ldots \ldots$ & -0.249 & 0.794 & -0.555 & 0.0 & 0.0 & 0.234 \\
\hline$w \ldots \ldots \ldots \ldots$ & 0.0 & -0.227 & 0.792 & -0.567 & 0.0 & 0.050 \\
\hline$x \ldots \ldots \ldots \ldots \ldots$ & 0.0 & 0.707 & -0.707 & 0.0 & 0.0 & -0.988 \\
\hline$y \ldots \ldots \ldots \ldots \ldots$ & 0.0 & 0.0 & -0.270 & 0.800 & -0.534 & 0.054 \\
\hline
\end{tabular}



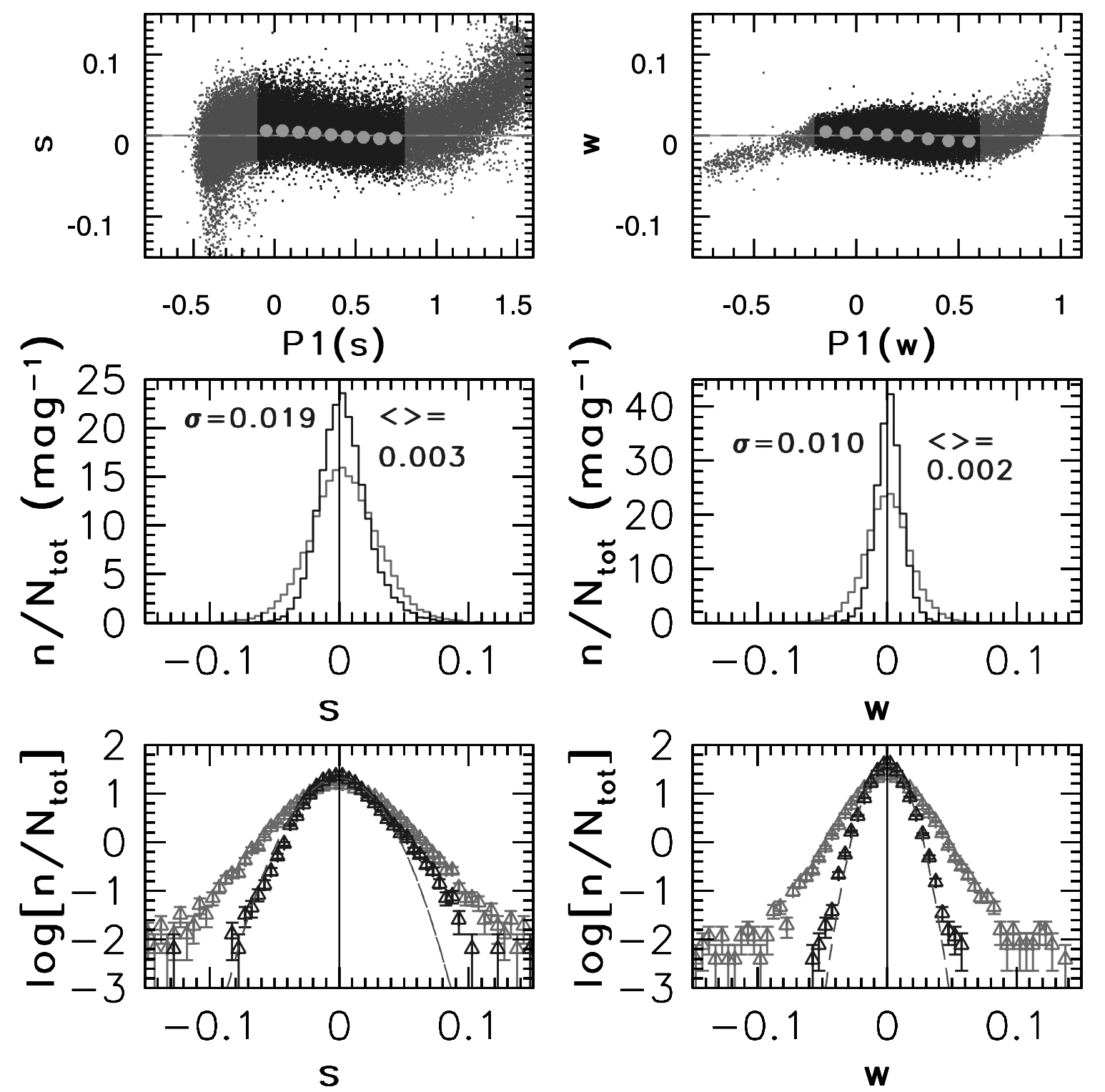

FIG. 8.-Top row: Two projections of the stellar locus (rotated locus from the ugr and gri planes; see Fig. 3) constructed using averaged photometry. The large dots show the median values of the $s$ and $w$ principal colors (perpendicular to the locus at its blue edge) in bins of the principal color along the locus. Middle and bottom rows: Histograms for each principal color on linear and logarithmic scales (essentially the locus cross sections). The dark histograms are constructed using the averaged photometry, and the light histograms are based on single-epoch photometry. The former are narrower than the latter due to increased photometric accuracy. The best-fit Gaussians, with parameters listed in the middle row, are shown by the dashed lines in the bottom row (only for the averaged photometry). [See the electronic edition of the Journal for a color version of this figure.]

conclusion is reached by comparing the averaged photometry with the secondary standard star network: the median photometric residuals at the so-called crossing colors ${ }^{38}$ are $4,6,3,2$, and 2 mmag in the ugriz bands, respectively. Yet another test is a direct comparison of averaged photometry with single-epoch photometry. Using the SDSS Data Release 5 photometry, we find that the largest median magnitude difference between the two sets is $2 \mathrm{mmag}$ in the $u$ band.

It is noteworthy that the widths of the principal color distributions (i.e., the thickness of the stellar locus) constructed with averaged photometry are much smaller than when using singleepoch data (see the bottom four panels in Fig. 8). Indeed, all four principal color distributions are "resolved" using this highquality photometry (see the fifth column in Table 3).

\footnotetext{
${ }^{38}$ Crossing colors are roughly the median colors of the observed stellar population; for details, see http://www.sdss.org/dr5/algorithms/jeg_photometric_ eq_dr1.html.
}

TABLE 3

The Effect of Repeated Measurements on the Width of the Stellar Locus

\begin{tabular}{|c|c|c|c|c|}
\hline $\mathrm{PC}$ & rms 1 Obs. ${ }^{a}$ & rms $N$ Obs. ${ }^{\mathrm{b}}$ & Median $^{\mathrm{c}}$ & $\begin{array}{c}\text { Width for } \\
\text { PC/PC Error }\end{array}$ \\
\hline$s \ldots \ldots \ldots \ldots \ldots$ & 31 & 19 & 3.0 & 3.02 \\
\hline$w \ldots \ldots \ldots \ldots \ldots \ldots$ & 25 & 10 & 1.1 & 1.82 \\
\hline$x \ldots \ldots \ldots \ldots \ldots$ & 42 & 34 & 1.2 & 5.34 \\
\hline$y \ldots \ldots \ldots \ldots \ldots$ & 23 & 9 & 0.8 & 1.64 \\
\hline
\end{tabular}

${ }^{a}$ The locus width determined using single-epoch SDSS observations (in millimagnitudes).

b The locus width determined using multiple SDSS observations (in millimagnitudes).

c The median principal color determined using multiple SDSS observations (in millimagnitudes).

d The locus width normalized by expected measurement errors. 

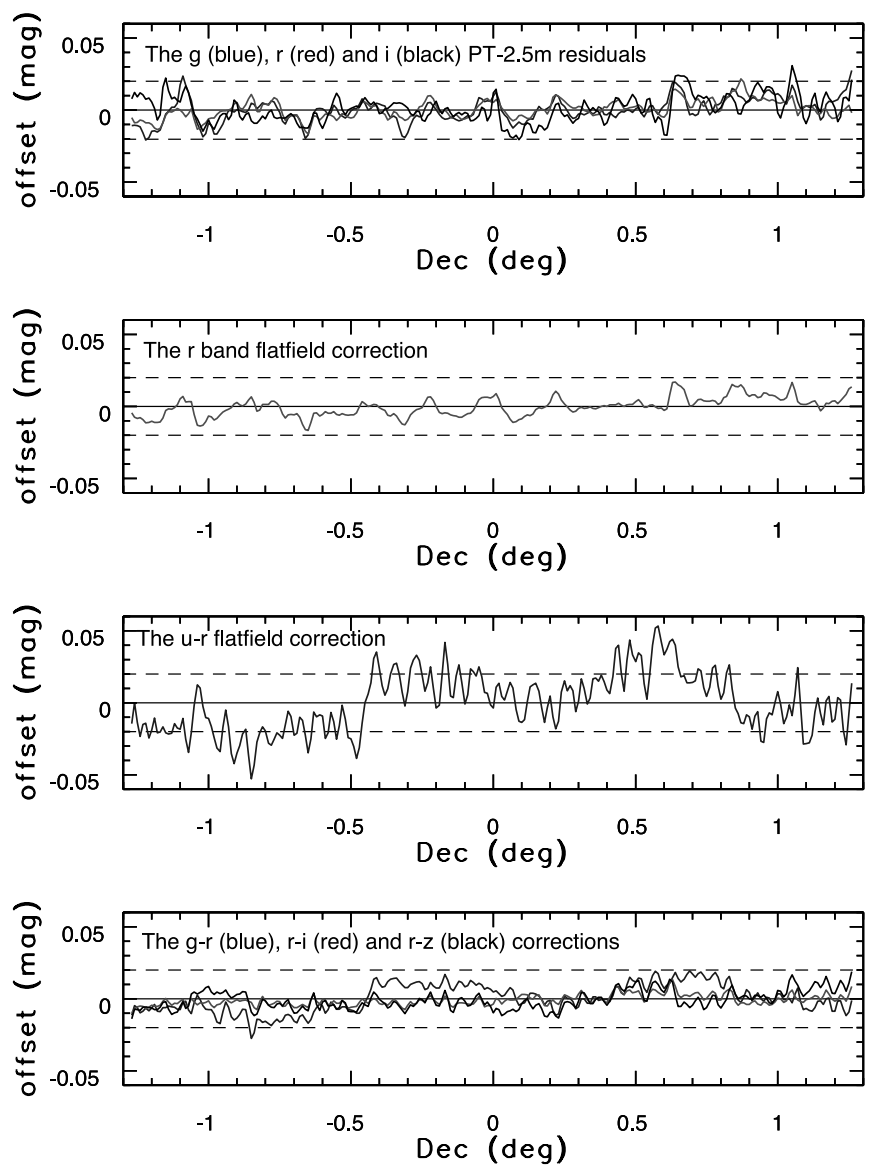

FIG. 9.-Top panel: Distribution of the residuals between the PT photometry and averaged magnitudes in the gri bands. Second panel: Applied flat-field correction in the $r$ band, which was derived as the mean of the residuals shown in the top panel. Third and fourth panels: Applied flat-field corrections in the other four bands, expressed relative to the $r$ band (third panel, $u$; fourth panel, giz), which were derived using the stellar locus colors. The low-order statistics for these corrections are listed in Table 4. [See the electronic edition of the Journal for a color version of this figure.]

Because the intrinsic widths of the principal color distributions are so small, principal colors can be used to efficiently track local calibration problems using a small number of stars, allowing a high spatial resolution. That is, we require that the locus not move in the multidimensional color space. In practice, the deviations of the principal colors from zero can be inverted, using an appropriate closure relation (see the next section), to yield flat-field corrections (Ivezic et al. 2004a). With bins $0.01^{\circ}$ wide in the declination direction, or $1^{\circ}$ wide in the right ascension direction, the flat-field corrections can be determined with an accuracy of 5 mmag or better.

\subsubsection{Gray Corrections from the Comparison with the Secondary Standard Star Network}

The main advantage of the stellar locus method is that it can constrain $\delta_{m r}$ with high spatial resolution. However, it is insensitive to gray errors, parameterized by $\delta_{r}$ (e.g., an overall gradient of photometric zero points in the declination direction that is the same in all five bands would have no effect on stellar colors). On the other hand, the PT comparison can constrain $\delta_{r}$, but it does not provide enough spatial resolution to derive flat-field corrections, especially in the $u$ and $z$ bands. Therefore, we combine these two methods to derive flat-field corrections $\delta_{m}^{\mathrm{ff}}$ (decl.).

The median differences between the averaged $2.5 \mathrm{~m}$ photometry and PT photometry for secondary standard stars in the gri
TABLE 4

The Flat-Field Corrections

\begin{tabular}{|c|c|c|c|}
\hline Band & Width $^{\mathrm{a}}$ & Minimum $^{\mathrm{b}}$ & Maximum $^{\mathrm{c}}$ \\
\hline ur & 22 & -53 & 53 \\
\hline$g r$ & 12 & -27 & 19 \\
\hline$r$ & 7 & -17 & 17 \\
\hline ri & 4 & -10 & 13 \\
\hline$r z$ & 7 & -14 & 19 \\
\hline
\end{tabular}

Note.-The $r$-band correction is determined using observations with the PT, and the ugiz corrections are determined using the stellar locus method $(\operatorname{see} \S \S 2.6 .1$ and 2.6.2).

${ }^{a}$ The rms scatter for applied flat-field corrections (in millimagnitudes)

${ }^{b}$ The minimum value of the applied correction (in millimagnitudes).

${ }^{c}$ The maximum value of the applied correction (in millimagnitudes).

bands are shown in Figure 9 (top panel). The median differences are computed for $0.01^{\circ}$ wide bins and then smoothed by a triangular filter $\left[y_{i}\right.$ is replaced by $\left.0.25\left(y_{i-1}+2 y_{i}+y_{i+1}\right)\right]$. The residuals in all three bands display similar behavior and imply about 0.02 peak-to-peak variation between the center and edges on each CCD (resulting in about $6 \mathrm{mmag}$ rms contribution to the overall errors), as well as an overall $0.01-0.02$ mag tilt. These systematic errors may be due to imperfect flat-field vectors used to reduce the data, incorrectly determined scattered-light correction (the two are somewhat coupled in the data reduction procedures), or problems in the PT itself (such as the PT flat field).

At face value, these residuals could be used to correct the averaged $2.5 \mathrm{~m}$ photometry in each band ( $g r i)$ separately. However, doing so introduces noise in the stellar principal colors of about $5 \mathrm{mmag}(\mathrm{rms})$ and suggests that the differences in photometric residuals between the three bands are dominated by PT measurement noise. On the other hand, the $2.5 \mathrm{~m}$ versus PT residuals do contain information about "gray" errors that cannot be determined using stellar locus. Hence, we take the mean value of the $2.5 \mathrm{~m}$ versus PT residuals in the $g r i$ bands to represent the $\delta_{r}$ flatfield correction and apply it to the averaged $2.5 \mathrm{~m}$ photometry in the $r$ band. The applied $r$-band correction is shown in Figure 9 (second panel) and has an rms scatter of $7 \mathrm{mmag}$ (for 250 bins), with the largest correction less than $0.02 \mathrm{mag}$.

In the second step, we use the stellar locus to derive the $\delta_{m r}$ corrections in each band (ugiz). The derivation of these corrections is essentially identical to the procedure described by Ivezić et al. (2004a). Also, together with a PT-based $\delta_{r}$ correction, this is essentially the same method as used to derive flat-field corrections for the whole SDSS survey. ${ }^{39}$ In particular, we used the same closure relation (the stellar locus method gives four equations for five unknowns) that is based on averaged $2.5 \mathrm{~m}$ versus PT residuals in the gri bands. The resulting flat-field corrections, $\delta_{m r}$, in the ugiz bands are shown in the third and fourth panels in Figure 9 and summarized in Table 4.

Due to low stellar counts and the strongest scattered light, the $u$-band correction is expected to have the largest noise $(\sim 5-$ $10 \mathrm{mmag}$ ), which is consistent with the observed behavior. It is thus likely that some of the variation on scales of $\sim 0.01^{\circ}$ is not real. On the other hand, it could be argued that systematic errors could actually be much larger on even smaller spatial scales but get averaged out in 90 pixel wide bins. However, in addition to not having a reason to believe in such high spatial frequency effects (e.g., the sky background does not show any evidence for them), no additional scatter, except the expected statistical noise, is observed when the bin size is decreased by a factor of 4 .

\footnotetext{
${ }^{39}$ For details, see http://www.sdss.org/dr5/algorithms/flatfield.html.
} 

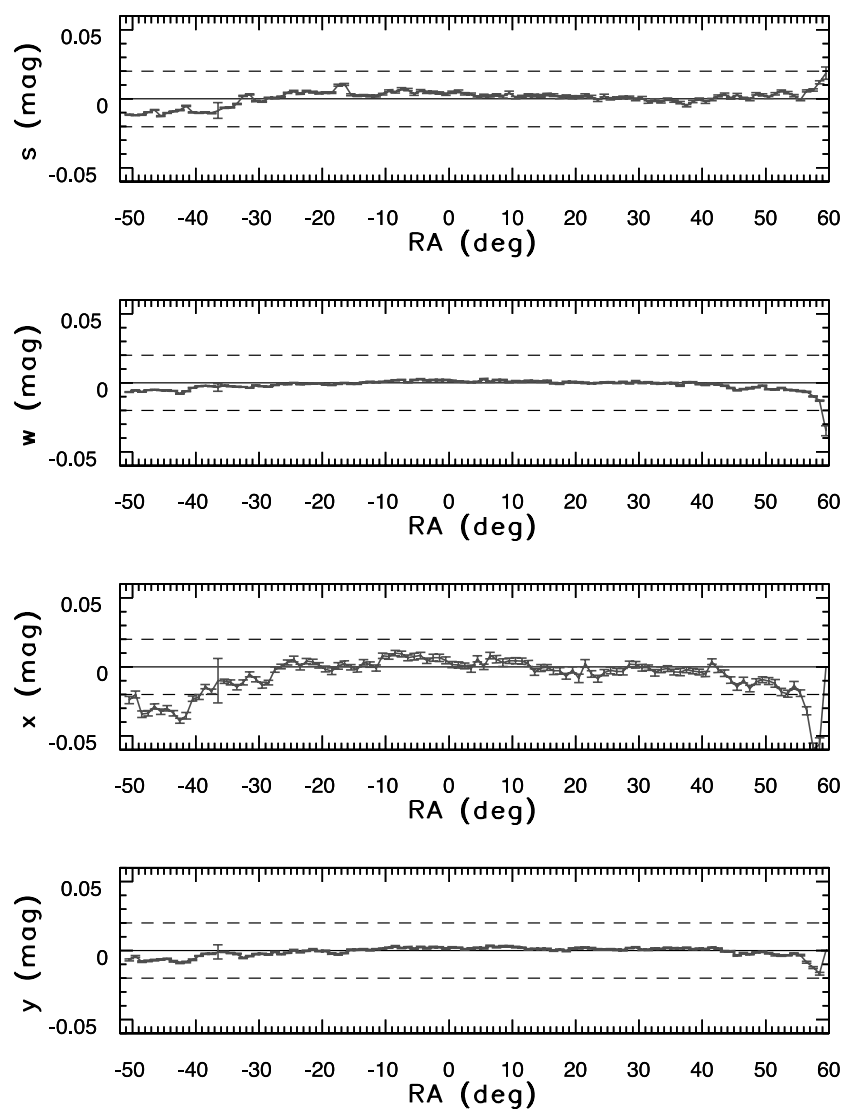

Fig. 10.-Dependence of the position of the stellar locus in ugriz color space, as parameterized by the median principal colors $s w x y$, as a function of right ascension. Close to the edges, the median colors deviate significantly from zero. This is caused by intrinsic changes in the stellar locus due to stellar population variations and overestimated interstellar extinction corrections for red stars, rather than calibration problems. [See the electronic edition of the Journal for a color version of this figure.]

Last but not least, it is important to emphasize that these corrections are not setting photometric zero points, but only correcting for variations in response across each CCD. As discussed above, the $\mathrm{AB}$ photometric zero points, relative to the fiducial $r$ band, are effectively set by adopting values for $\Delta_{m r}$.

\subsection{Tests of Catalog Quality}

\subsubsection{The Internal Tests}

At least in principle, the same methods used to derive $\delta_{m}^{\mathrm{ff}}$ (decl.) could be used to derive $\delta_{m}^{\text {ext }}$ (R.A.). However, in practice this is not possible for at least two reasons: first, the right ascension distribution of secondary standard stars is not as uniform as their declination distribution, and second, the assumption of the constancy of the stellar locus in color space is invalid along the "long" scan direction (discussed below). For these reasons, we only use the PT comparison and stellar locus methods to estimate the level of internal zero-point variations with right ascension, and we do not correct the data. In the next section we also use another method, based on galaxy colors, as an independent test of catalog integrity.

Figure 10 shows the median principal colors in bins of right ascension. It is evident that the principal colors are close to zero for right ascension in the range $-25^{\circ}<$ R.A. $<40^{\circ}$ but outside this range deviate significantly from zero. This does not necessarily indicate problems with photometric calibration, because the stellar locus method is expected to fail at low Galactic latitudes for
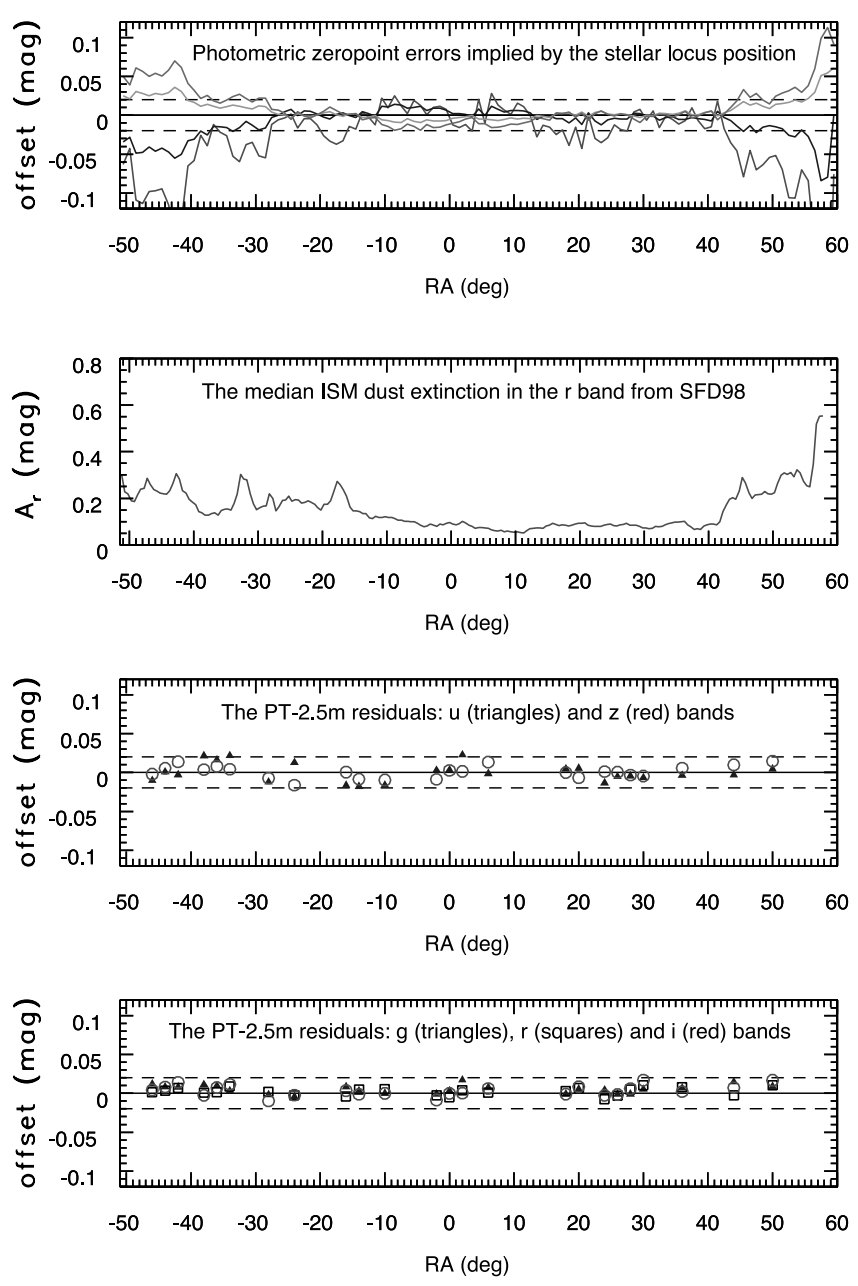

FIG. 11.-Top panel: Implied photometric zero-point errors based on the stellar locus method (ugiz from the bottom to the top at either edge). While the implied errors are small for $-25^{\circ}<$ R.A. $<40^{\circ}$, they become exceedingly large outside this range. This is due to problems with the stellar locus method rather than problems with calibration. Second panel: Median $r$-band extinction derived from the SFD98 maps. Third and fourth panels: Median residuals between the PT photometry and averaged magnitudes in the $u z$ (third panel) and gri bands ( fourth panel). The low-order statistics for these residuals are listed in Table 5. [See the electronic edition of the Journal for a color version of this figure.]

several reasons. First, the mean metallicity of stars increases at low Galactic latitudes, and this change may affect the $s$ and $w$ colors. Second, at low latitudes red dwarfs are not behind the entire dust screen measured by the SFD98 maps (see Jurić et al. [2005] for a discussion of this point), and thus the $x$ color will be biased blue (i.e., the colors of red dwarfs are overcorrected for the ISM reddening). And third, at low latitudes the dust column increases quickly (see Fig. 11), and even small errors in the assumed wavelength dependence of the dust extinction, or the extinction itself as given by the SFD98 maps, will have noticeable effects on the principal colors. For these reasons it seems plausible that the deviations seen in Figure 10 are not dominated by zero-point errors.

This conclusion is supported by direct comparison of the averaged and PT photometry (Fig. 11). For example, the largest median photometric residual between the averaged catalog and PT observations in the $u$ band is $\sim 0.02 \mathrm{mag}$ (see Table 5), which is much smaller than the $0.1 \mathrm{mag}$ discrepancy implied by the stellar locus method.

Table 5 shows that the rms scatter of the median photometric residuals (evaluated in $2^{\circ}$ wide bins in the right ascension direction) 
TABLE 5

The Statistics of the Median PT 2.5 m Residuals

\begin{tabular}{|c|c|c|c|c|c|c|}
\hline Band & Residuals $^{\mathrm{a}}$ & Width $^{\mathrm{b}}$ & Minimum $^{c}$ & Maximum $^{\mathrm{d}}$ & $N^{\mathrm{e}}$ & Residuals $^{f}$ \\
\hline$u \ldots \ldots \ldots \ldots . .$. & -2 & 9 & -18 & 23 & 175 & 5 \\
\hline$g \ldots \ldots \ldots \ldots$ & 6 & 7 & -4 & 17 & 647 & 5 \\
\hline$r \ldots \ldots \ldots \ldots$ & 3 & 7 & -7 & 10 & 627 & 3 \\
\hline$i \ldots \ldots \ldots \ldots$ & 4 & 7 & -10 & 17 & 621 & 2 \\
\hline$z \ldots \ldots \ldots \ldots$ & 1 & 8 & -16 & 15 & 286 & -2 \\
\hline
\end{tabular}

${ }^{a}$ The median value for the bin medians (in millimagnitudes). There are 24 bins, distributed inhomogeneously in the right ascension direction.

${ }^{b}$ The rms scatter for the bin medians (in millimagnitudes).

c The minimum value for the median residuals (in millimagnitudes).

d The maximum value for the median residuals (in millimagnitudes).

e The median number of stars per bin.

${ }^{\mathrm{f}}$ The median value of the residuals for stars with colors within 0.02 mag of the crossing colors (in millimagnitudes).

between the averaged catalog and PT observations is $<0.01 \mathrm{mag}$ in all five bands. Some of that scatter must come from the PT data themselves, and thus the true scatter of the photometric zero points in the averaged catalog is probably even smaller than that listed in Table 5. In addition, Table 5 shows that the averaged catalog and PT measurements are on the same system to within a few millimagnitudes (using the recommended photometric transformations between the two telescopes listed at the SDSS Web site; see $\S 2.2$ ).

We have also compared the catalog presented here to the photometric reductions described by Padmanabhan et al. (2007). As discussed in $\S 1$, they determined flat fields by minimizing the errors in relative photometry of multiply observed stars over the whole survey region. Hence, this comparison is an essentially independent test of the flat-field corrections derived here, despite the fact that both catalogs are based on the same observations. We bin the photometric differences between the catalogs in $0.01^{\circ}$ wide declination bins and compute the median residual for each bin and band. The median values of these medians represent zero-point offsets in each band and are equal to $-7,2,-1,2$, and $5 \mathrm{mmag}$ in the ugriz bands, respectively. The rms scatter of the median residuals reflects systematic errors due to flat-field errors, and we measure 27, 6, 5, 6, and $8 \mathrm{mmag}$ in the ugriz bands, respectively. Except in the $u$ band, these values indicate that the systematic flat-field errors are very small. In the $u$ band, Padmanabhan et al. (2007) expect errors of about $0.01 \mathrm{mag}$, and the distribution width of the $s$ color implies about $0.01 \mathrm{mag}$ for the catalog discussed here, predicting about 14 mmag instead of the measured $27 \mathrm{mmag}$. It is plausible that the $u$-band photometry may contain systematic errors unrecognized by any of the methods discussed here.

\subsubsection{The Tests of Catalog Quality Based on Galaxy Colors}

The color distribution of galaxies is bimodal (Strateva et al. 2001; Yasuda et al. 2001; Baldry et al. 2004). Red galaxies have an especially tight color-redshift relation (Eisenstein et al. 2001), with an rms of $0.12 \mathrm{mag}$ for the $u-g$ color, $0.05 \mathrm{mag}$ for the $g-r$ color, and $0.03 \mathrm{mag}$ for the $r-i$ and $i-z$ colors (using model magnitudes). Deviations from the mean relations can thus be used to track local calibration problems. Of course, since this is a colorbased method, it can only constrain $\delta_{m r}$, and, because red galaxies are faint in the $u$ band, cannot achieve high spatial resolution in this band. Nevertheless, it is a useful addition to the stellar locus method because it is independent of the Milky Way structure and secondary star network (although it is sensitive to errors in the ISM dust extinction correction).
TABLE 6

Residuals from the Mean Color-Redshift Relation for Red Galaxies

\begin{tabular}{ccccc}
\hline \hline Color & rms $(\text { Decl. })^{\mathrm{a}}$ & $\chi$ (Decl.) & rms (R.A.) & $\chi$ (R.A.) \\
\hline$u-g \ldots \ldots \ldots$. & 21 & 1.5 & 18 & 1.3 \\
$g-r \ldots \ldots \ldots$. & 4 & 1.3 & 12 & 2.6 \\
$r-i \ldots \ldots \ldots \ldots$ & 3 & 1.3 & 6 & 3.5 \\
$i-z \ldots \ldots \ldots \ldots$. & 9 & 2.9 & 6 & 2.9 \\
\hline
\end{tabular}

NOTE.-The rms widths of color-residual distributions (in millimagnitudes) and the widths of distributions of residuals normalized by statistical noise $(\chi)$, using mean color-redshift relations (see text).

${ }^{\mathrm{a}}$ The rms for the declination direction, using $0.025^{\circ}$ wide bins $\left(0.1^{\circ}\right.$ for the $u-g$ color). color).

The rms for the right ascension direction, using $2^{\circ}$ wide bins $\left(5^{\circ}\right.$ for the $u-g$

We select 19,377 red galaxies with SDSS spectra from the redshift range $0.02-0.36$ using an empirical condition,

$$
0<(g-r)-0.6-2.75 \times \text { redshift }<0.3,
$$

and determine their median colors as a function of redshift using 0.01 wide redshift bins. The residuals from the median colorredshift relation are then binned by declination to constrain $\delta_{m}^{\mathrm{ff}}$ and by right ascension to constrain $\delta_{m}^{\text {ext }}$. The rms for color residuals and the widths of distributions of residuals normalized by statistical noise (based on quoted photometric errors) are listed in Table 6.

The residuals binned in the declination direction are generally small and consistent with statistical noise. The largest deviations from zero are seen for the $i-z$ color, with an rms of 9 mmag and maximum deviation of $17 \mathrm{mmag}$ (see Fig. 12, top). Although the rms is fairly small, it is a factor of 2.9 larger than the expected noise. The shape of the $i-z$ residuals is similar to that of the $i-z$ residuals for stars discussed in $\S 2.5 .2$ and shown in Figure 7 (bottom). The rms scatter for the difference between the stellar and galaxy $i-z$ residuals, shown in Figure 12 (bottom), is 6 mmag. Since the rms for the galaxy residuals is larger $(9 \mathrm{mmag})$, it is plausible
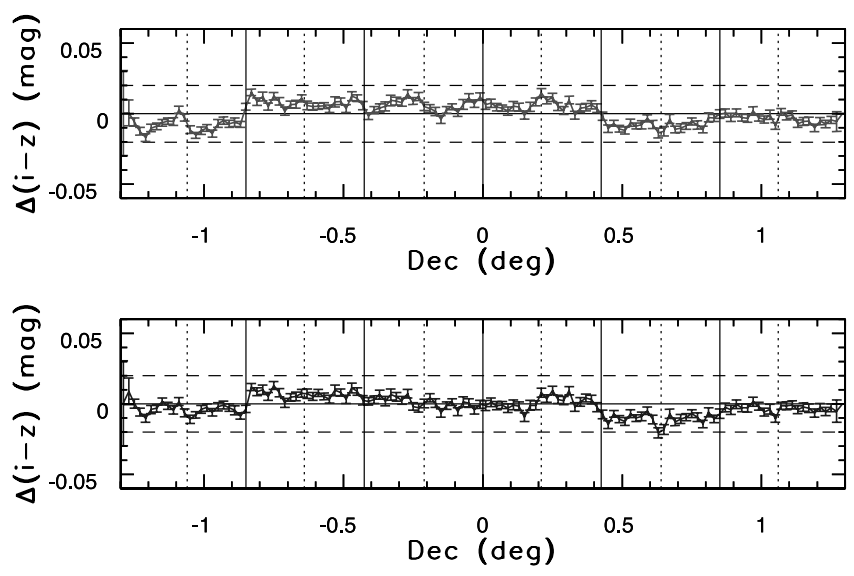

FIG. 12.-Top: Dependence of the median $i-z$ color residuals (with respect to a mean color-redshift relation) for red galaxies as a function of declination. The vertical solid lines mark the approximate boundaries between different camera columns, with the vertical dashed lines marking approximate boundaries between the "north" and "south" strips in a stripe. The small rms scatter of only 9 mmag demonstrates that flat-field corrections based on the stellar locus position in color space are also applicable for galaxies. Bottom: Difference between the values shown in the top panel and the curve shown in the bottom panel of Fig. 7. The rms scatter for the residuals is $6 \mathrm{mmag}$. [See the electronic edition of the Journal for a color version of this figure.] 

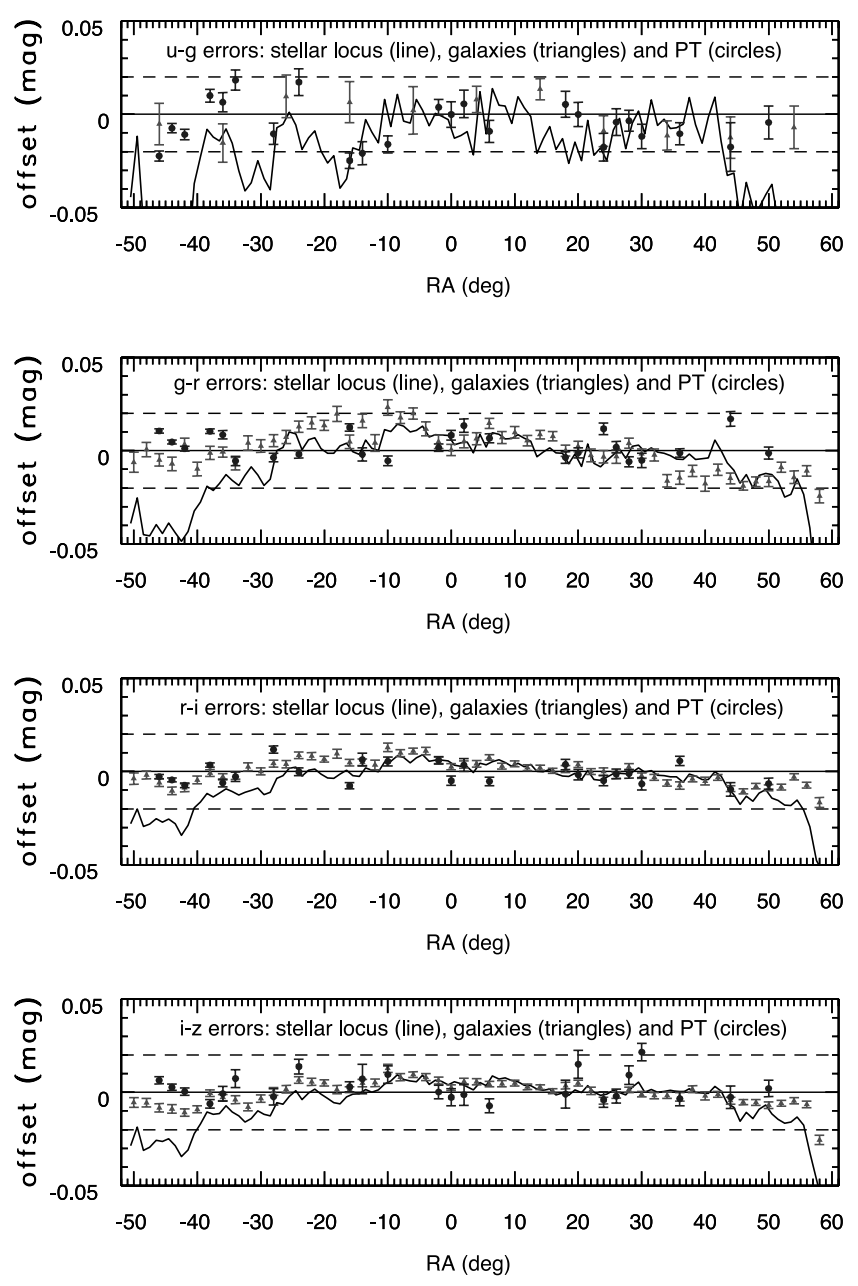

FIG. 13.-Comparison of systematic color errors implied by different methods. Note that the errors implied by the stellar locus method (jagged line) become very large outside the $-25^{\circ}<\alpha_{\mathrm{J} 2000.0}<40^{\circ}$ range. As galaxy colors (triangles) and a direct comparison with the SDSS secondary standard star network (circles) suggest, this is due to problems with the stellar locus method rather than problems with calibration. [See the electronic edition of the Journal for a color version of this figure.]

that the same systematic effect dominates the remaining photometric errors for both stars and galaxies. While it is not clear what the cause of this (small) effect is, a plausible explanation is the dependence of flat fields on source color. ${ }^{40}$

The results for binning in the right ascension direction are shown in Figure 13, where we compare different methods. The rms for implied color errors (with $2^{\circ}$ wide bins) from galaxies is 0.006 mag for the $r-i$ and $i-z$ colors, 0.012 mag for the $g-r$ color, and $0.018 \mathrm{mag}$ for the $u-g$ color. The overall behavior of red galaxy color residuals agrees better with the PT method than with color errors implied by the stellar locus method. In particular, the large errors outside the $-25^{\circ}<$ R.A. $<40^{\circ}$ range implied by the latter method are not consistent with red galaxy color residuals. On the other hand, both red galaxy colors and the stellar locus seem to show a trend that the colors are redder around R.A. $=-10^{\circ}$ than around R.A. $=40^{\circ}$. The amplitude of this effect varies from about 0.02 mag for the $g-r$ color to about 0.01 mag for the $i-z$ color, while the upper limit on such a slope implied by the PT comparison is $<0.01 \mathrm{mag}$.

\footnotetext{
${ }^{40}$ It is fair to ask whether the applied flat-field corrections, derived from stellar colors, are actually appropriate for galaxies. They are, since the $i-z$ color residuals for galaxies without any flat-field corrections are about twice as large as those shown in Fig. 12 (top).
}

It is not clear what the cause of this discrepancy is. The obvious culprit is the correction for interstellar dust extinction, but the implied deviation is too large to be explained by any plausible errors in the SFD98 maps. As shown in Figure 11, the median extinction in the $r$ band for the $-10^{\circ}<$ R.A. $<40^{\circ}$ range is below $0.1 \mathrm{mag}$, and the resulting median correction for, e.g., the $g-r$ color is below $0.04 \mathrm{mag}$. Hence, to induce a $0.02 \mathrm{mag}$ trend in the $g-r$ color, the SFD98 value for the $r$-band extinction, $A_{r}$, would have to be in error by $0.05 \mathrm{mag}$ (the difference between the values provided for R.A. $=-10^{\circ}$ and R.A. $=40^{\circ}$ ). This implies relative errors for the SFD98 maps in the range from 50\% (if $A_{r}$ at R.A. $=$ $-10^{\circ}$ is underestimated) to $100 \%$ (if $A_{r}$ at R.A. $=40^{\circ}$ is overestimated), which seems unlikely (although not impossible).

We conclude that the PT comparison provides a good estimate of the remaining zero-point errors in the catalog, as listed in Table 5, but caution that we do not understand the above systematic behavior of the stellar and galaxy colors, and that only the PT constrains possible gray errors. In the next section we discuss a comparison to an external data set that supports this conclusion.

\subsubsection{An External Test of Catalog Quality Based on Stetson's Standards}

The only large external data set with sufficient overlap, depth, and accuracy to test the quality of the stripe 82 catalog is that provided by Stetson $(2000,2005)$. Stetson's catalog lists photometry in the BVRI bands (Stetson's photometry is tied to Landolt's standards) for $\sim 1200$ stars in common (most have $V<19.5$ ). We synthesize the BVRI photometry from the SDSS gri measurements using photometric transformations of the following form:

$$
m_{\text {Stetson }}-m_{\mathrm{SDSS}}=A c^{3}+B c^{2}+C c+D
$$

where $m_{\text {Stetson }}=(B V R I), m_{\mathrm{SDSS}}=(g, g, r, i)$, and the color $c$ is measured by SDSS ( $g-r$ for the $B$ and $V$ transformations and $r-i$ for the $R$ and $I$ transformations). The measurements are not corrected for ISM reddening. Traditionally, such transformations are assumed to be linear in color. ${ }^{41}$ We use higher order terms in equation (10) because at the $1 \%-2 \%$ level there are easily detectable deviations from linearity for all color choices (for details and plots, see Ivezić et al. 2007). The best-fit coefficients for the transformation of SDSS gri measurements to the BVRI system ${ }^{42}$ and low-order statistics for the $m_{\text {Stetson }}-m_{\text {SDSS }}$ difference distribution $^{43}$ are listed in Table 7. We find no trends as a function of magnitude at the $<0.005$ mag level. With the listed transformations, the SDSS catalog described here could also be used to calibrate the data to the BVRI system with a negligible loss of accuracy due to transformations between the two systems.

The $B V R I$ photometry from Stetson and that synthesized from SDSS agree at the level of $0.02 \mathrm{mag}$ (rms scatter for the magnitude differences of individual stars; note that the systems are tied to each other to within a few millimagnitudes by the transformations listed in Table 7). This scatter is consistent with the claimed accuracy of both catalogs (the magnitude differences normalized by the implied error bars are well described by Gaussians with widths in the range $0.7-0.8$ ). This small scatter allows us to test

\footnotetext{
41 For various photometric transformations between the SDSS and other systems, see Abazajian et al. (2005) and http://www.sdss.org/dr5/algorithms/ sdssUBVRITransform.html.

42 The same transformations can be readily used to transform measurements in the $B V R I$ system to the corresponding gri values because $B-V=f(g-r)$ and $R-I=f(r-i)$ are monotonic functions.

43 Note that these transformations are valid only for main-sequence stars with colors in the range $g-r>0.2$ and $r-i<1.5$ (roughly, $0.3<B-V<1.6$ ). Extrapolation outside this range may result in large errors.
} 
TABLE 7

Comparison with Stetson's Standards: gri-to-BVRI Transformations

\begin{tabular}{|c|c|c|c|c|c|c|c|c|c|}
\hline Color & Residuals $_{\text {med }}{ }^{a}$ & $\sigma_{\text {med }}^{\mathrm{b}}$ & $\chi_{\text {med }}{ }^{\mathrm{c}}$ & Residuals $_{\text {all }}{ }^{\mathrm{d}}$ & $\sigma_{\text {all }}^{\mathrm{e}}$ & $A^{\mathrm{f}}$ & $B^{\mathrm{f}}$ & $C^{\mathrm{f}}$ & $D^{\mathrm{f}}$ \\
\hline$B-g$ & -1.6 & 8.7 & 1.4 & 1.0 & 32 & 0.2628 & -0.7952 & 1.0544 & 0.0268 \\
\hline$V-g$ & 0.8 & 3.9 & 1.0 & 0.9 & 18 & 0.0688 & -0.2056 & -0.3838 & -0.0534 \\
\hline$R-r \ldots \ldots \ldots \ldots \ldots \ldots \ldots$ & -0.1 & 5.8 & 0.9 & 1.2 & 15 & -0.0107 & 0.0050 & -0.2689 & -0.1540 \\
\hline$I-i$ & 0.9 & 6.1 & 1.0 & 1.2 & 19 & -0.0307 & 0.1163 & -0.3341 & -0.3584 \\
\hline
\end{tabular}

Notes.-These transformations are valid only for main-sequence stars with colors in the range $g-r>0.2$ and $r-i<1.5$ (roughly, $0.3<B-V<1.6$ ). Extrapolation outside this range may result in large ( $0.1 \mathrm{mag}$ or larger for hot white dwarfs) errors.

${ }^{a}$ The median value of median transformation residuals (differences between the measured values of colors listed in the first column and those synthesized using eq. [10]) in 0.1 mag wide $g-r$ bins for stars with $0.25<g-r<1.45$ (in millimagnitudes). These medians of medians measure the typical level of systematics in the gri-to- $B V R I$ photometric transformations introduced by the adopted analytic form (see eq. [10]).

b The rms scatter for median residuals described above (in millimagnitudes).

${ }^{c}$ The rms scatter for residuals normalized by statistical noise. The listed values are $\sim 1$, which indicates that the scatter around adopted photometric transformations discussed in footnote a is consistent with expected noise.

${ }^{\mathrm{d}}$ The median value of residuals evaluated for all stars (in millimagnitudes).

e The rms scatter for residuals evaluated for all stars (in millimagnitudes).

${ }^{f}$ Coefficients $A-D$ needed to transform SDSS photometry to the $B V R I$ system (see eq. [10])

for the spatial variation of zero points between the two data sets, despite the relatively small number of stars in common.

Stars in common are found in four isolated regions that coincide with the historical and well-known Kapteyn selected areas $113,92,95$, and 113. We determine the zero-point offsets between the SDSS and Stetson's photometry for each region separately by synthesizing $B V R I$ magnitudes from SDSS gri photometry and comparing them to Stetson's measurements. The implied zeropoint errors (which, of course, can be due to either the SDSS or the Stetson data set, or both) are listed in Table 8. For regions 1-3 the implied errors are only a few millimagnitudes (except for the $B-g$ color in region 1). The discrepancies are much larger for the three red colors in region 4. A comparison with the results of internal SDSS tests described in $\S \S 2.7 .1$ and 2.7.2 suggests that these discrepancies are more likely due to zero-point offsets in Stetson's photometry for this particular region than to problems with SDSS photometry. We contacted P. Stetson, who confirmed that his observing logs were consistent with this conclusion. Only a small fraction of stars from Stetson's list are found in this region.

Given the results presented in this section, we conclude ${ }^{44}$ that the rms for the spatial variation of zero points in the SDSS stripe 82 catalog is below $0.01 \mathrm{mag}$ in the gri bands.

\section{THE UTILITY OF THE SDSS STRIPE 82 STANDARD STAR CATALOG}

As examples of the use of the standard star catalog, we discuss the calibration of data obtained in nonphotometric conditions and

\footnotetext{
${ }^{44}$ Here we assumed that it is a priori unlikely that the SDSS and Stetson's zeropoint errors are spatially correlated.
}

a detailed and robust measurement of the morphology of the stellar locus in color-color space.

\subsection{Calibration of Nonphotometric Data}

The existence of a technique to photometrically calibrate nonphotometric data would greatly increase the efficiency of telescopes. As one particular example of how our catalog can support a large project, consider the SDSS-II supernova survey (Sako et al. 2005). This survey aims to obtain repeat images of stripe 82 with a sufficient cadence to enable the discovery of new Type Ia supernovae. This requirement sometimes results in observations obtained through clouds with several magnitudes of extinction. In such highly nonphotometric conditions the standard photometric calibration described in $\S 2.2$ fails because the fields with standard stars are too sparsely distributed to be able to resolve fast variations in cloud extinction.

\subsubsection{A Method to Track Fast Cloud Extinction Variations}

Due to its high stellar density, the standard star catalog described in this paper can be used for calibration of data obtained in grossly nonphotometric conditions. The typical number of calibration stars in each SDSS field $\left(9 \times 13 \operatorname{arcmin}^{2}\right)$ at high Galactic latitudes is $10-15$ in the $u$ band, $40-50$ in the gri bands, and 3040 in the $z$ band. Based on tests of several nonphotometric SDSS-II runs, it was found that the cloud extinction variations can be tracked with a sufficient temporal resolution $(\sim 3 \mathrm{~s})$ to obtain photometric zero-point accuracy comparable to that characteristic for photometric nights $(1 \%-2 \%$ in gri and $2 \%-3 \%$ in $u$ and $z$; Ivezić et al. 2004a).

TABLE 8

Comparison with Stetson's Standards: Photometric Zero-Point Variations

\begin{tabular}{|c|c|c|c|c|c|c|c|c|c|c|c|c|}
\hline Color & Residuals $_{\mathrm{R} 1}{ }^{\mathrm{a}}$ & $\sigma_{\mathrm{R} 1}{ }^{\mathrm{b}}$ & $N_{\mathrm{R} 1}^{\mathrm{c}}$ & Residuals $_{\mathrm{R} 2}{ }^{a}$ & $\sigma_{\mathrm{R} 2}^{\mathrm{b}}$ & $N_{\mathrm{R} 2}{ }^{\mathrm{c}}$ & Residuals $_{R 3}{ }^{a}$ & $\sigma_{\mathrm{R} 3}{ }^{\mathrm{b}}$ & $N_{\mathrm{R} 3}{ }^{\mathrm{c}}$ & Residuals $_{\mathrm{R} 4}{ }^{\mathrm{a}}$ & $\sigma_{\mathrm{R} 4}{ }^{\mathrm{b}}$ & $N_{\mathrm{R} 4}{ }^{\mathrm{c}}$ \\
\hline$B-g \ldots \ldots \ldots$ & -29 & 21 & 92 & 6 & 27 & 165 & 8 & 42 & 155 & -4 & 27 & 281 \\
\hline$V-g \ldots \ldots \ldots$ & 0 & 17 & 99 & 0 & 15 & 217 & 6 & 25 & 161 & 17 & 19 & 282 \\
\hline$R-r \ldots \ldots \ldots \ldots$ & -6 & 16 & 58 & 4 & 16 & 135 & -8 & 12 & 11 & 39 & 27 & 60 \\
\hline$I-i \ldots \ldots \ldots \ldots$ & -11 & 16 & 94 & 6 & 18 & 205 & 2 & 16 & 124 & 19 & 15 & 47 \\
\hline
\end{tabular}

${ }^{\text {a }}$ The median value of residuals (in millimagnitudes) for transformations listed in the first column, evaluated separately for regions $1-4$, defined as follows: R1, R.A. $\sim 325$, decl. $<0$; R2, R.A. $\sim 15$; R3, R.A. $\sim 55$; R4, R.A. $\sim 325$, decl. $>0$.

b The rms scatter for the transformation residuals (in millimagnitudes).

c The number of stars in each region with good photometry in the required bands. 
The calibration is done in two steps. First, the implied zero points (whose variation is dominated by cloud extinction), $z p$, defined by

$$
m_{\text {calibrated }}=-2.5 \log (\text { counts })+z p,
$$

are computed for each star and median-filtered in time using a window with five stars in order to avoid outliers. Note that we assume that the clouds are gray and do not allow for color terms, an assumption which is justified a posteriori (see $\S 3.1 .4)$. In the second step, zero points are evaluated for each 2048 pixel wide (cross-scan direction) and 100 pixel long (in-scan direction) image segment, hereafter called a "calibration patch" (not to be confused with secondary star patches, discussed in $\S 2.2$ ). That is, a calibration patch is an $\sim 9 \operatorname{arcmin}^{2}$ large rectangle with an aspect ratio of $1: 20$, and the zero points are evaluated every $2.6 \mathrm{~s}$ (but note that the variations are smoothed out by the $54 \mathrm{~s}$ long exposure time).

The patch is much narrower in the in-scan direction because tests have shown that zero-point gradients across a field are much larger, by a factor of 10-50, in this direction (see Fig. 14). Consider three stars: star A; star B, which is, say, 25' (the column-tocolumn separation) away from star A in the scan direction; and star C, which is $25^{\prime}$ away from star A in the cross-scan direction. Stars $\mathrm{A}$ and $\mathrm{C}$ are observed at the same time, and the difference in their implied zero points measures the structure function (SF) of cloud opacity on a $25^{\prime}$ spatial scale. This is true irrespective of the cloud motion relative to the boresight. Here the SF of cloud opacity is defined as the rms width of the distribution of zero-point differences evaluated for pairs of points separated by some distance.

On the other hand, stars A and B are observed at times that differ by 1.7 minutes. If the component of the cloud angular velocity on the sky relative to the boresight and parallel to the scanning direction is $\omega^{\circ}$ minute $^{-1}$, the zero-point difference for stars $A$ and B samples the cloud structure on spatial scales of $25^{\prime}\left(\omega / \omega_{s}\right)$, where $\omega_{s}=0.25^{\circ}$ minute $^{-1}$ is the sidereal scanning rate (for simplicity, we assumed $\omega \gg \omega_{s}$, which is supported by the data). The observed behavior of zero points, such as that shown in Figure 14 , implies wind velocity in the range ${ }^{45} \omega=3^{\circ}-15^{\circ}$ minute $^{-1}$, or $\omega / \omega_{s} \sim 12-60$. Hence, drift scanning has the unfortunate property that the motion of an inhomogeneous extinction screen with a speed much larger than the sidereal scanning rate greatly magnifies the effective zero-point variations in the scan direction.

The zero points for each calibration patch are computed by taking all the stars from the patch, or finding the closest three stars for sparsely populated patches, and adopting the median value of their zero points. This is certainly not the only, nor perhaps the ideal, approach to calibrate patches, but we found that it works well in practice. The zero-point error is evaluated from the rms scatter of $z p$ evaluated for each calibration star, divided by the square root of the number of stars. We now discuss the performance of this method.

\subsubsection{Performance and Quality Tests}

The top panel in Figure 15 summarizes the behavior of cloud extinction in the $r$ band, as measured by the zero point $z p$ discussed above, for an SDSS-II supernova run (5646) obtained in strongly nonphotometric conditions. Although the cloud extinction during the first 90 minutes (corresponding to 150 SDSS fields)

\footnotetext{
45 This range is equivalent to angular speeds of up to half of the Moon's diameter per second. The plausibility of this wind velocity range was verified in extensive visual observations of the full Moon during frequent grossly nonphotometric nights in Seattle.
}

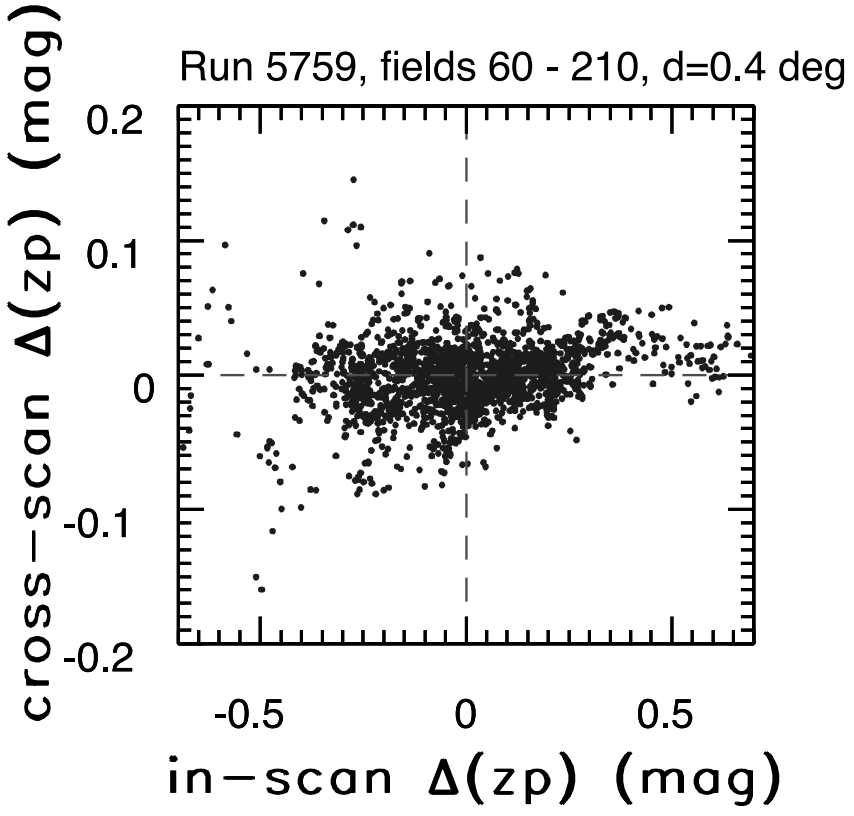

FIG. 14.-Comparison of cloud extinction gradients in the in-scan (right ascension, horizontal axis) and cross-scan (declination, vertical axis) directions for SDSS run 5759 , on a spatial scale of $\sim 0.4^{\circ}$ (each point is derived using zero points from three calibration patches). Note the different axis scales. For this particular run, the distribution width is 9.6 times larger for the in-scan than for the cross-scan direction. This is a consequence of cloud motion relative to the boresight and the driftscanning technique. [See the electronic edition of the Journal for a color version of this figure.]

varies between 0 and $\sim 6 \mathrm{mag}$, it is possible to robustly calibrate these data. Figure 16 zooms in on an 8 minute stretch of the same data where the cloud extinction varies between 0 and $\sim 3$ mag, with changes as fast as $0.05 \mathrm{mag} \mathrm{s}^{-1}$ (almost 2 mag per SDSS field). As shown in the figure (third row, left panel), the residuals have a distribution width of only 0.07 mag. The right panels in the third rows of Figures 15 and 16 demonstrate that most of this scatter is contributed by random photometric errors (i.e., errors in extracted source counts), rather than by calibration errors (large cloud extinction results in a smaller number of calibration stars, as well as in a lower signal-to-noise ratio for those calibration stars that are detected). Even with such a large and rapidly varying cloud extinction, the zero-point errors are smaller than 0.05 mag, with a median value of less than $0.02 \mathrm{mag}$. An example of a run with somewhat thinner and much more stable cloud cover is shown in Figure 17.

The calibration performance in other bands is similar. For example, although the number of calibration stars is smaller in the $u$ band than in the $r$ band, the median zero-point error for the same stretch of data as shown in Figure 17 is still only $0.01 \mathrm{mag}$, as illustrated in Figure 18.

\subsubsection{The Summary of Calibration Accuracy}

A summary of the final zero-point errors as a function of cloud extinction and band for one of the worst runs is shown in the left column of Figure 19. As the figure shows, the data can be calibrated with small zero-point errors even for such a bad case. Typically, the zero-point errors for the same cloud extinction are about twice as small as in this run. A calibration summary for a run with optically thick but exceptionally smooth clouds is shown in the right column of Figure 19. Overall, for cloud extinction of X mag, the zero-point uncertainty is typically smaller than (0.02-0.05)X for 95\% of the calibration patches, with a median of $(0.01-0.02) X$. 

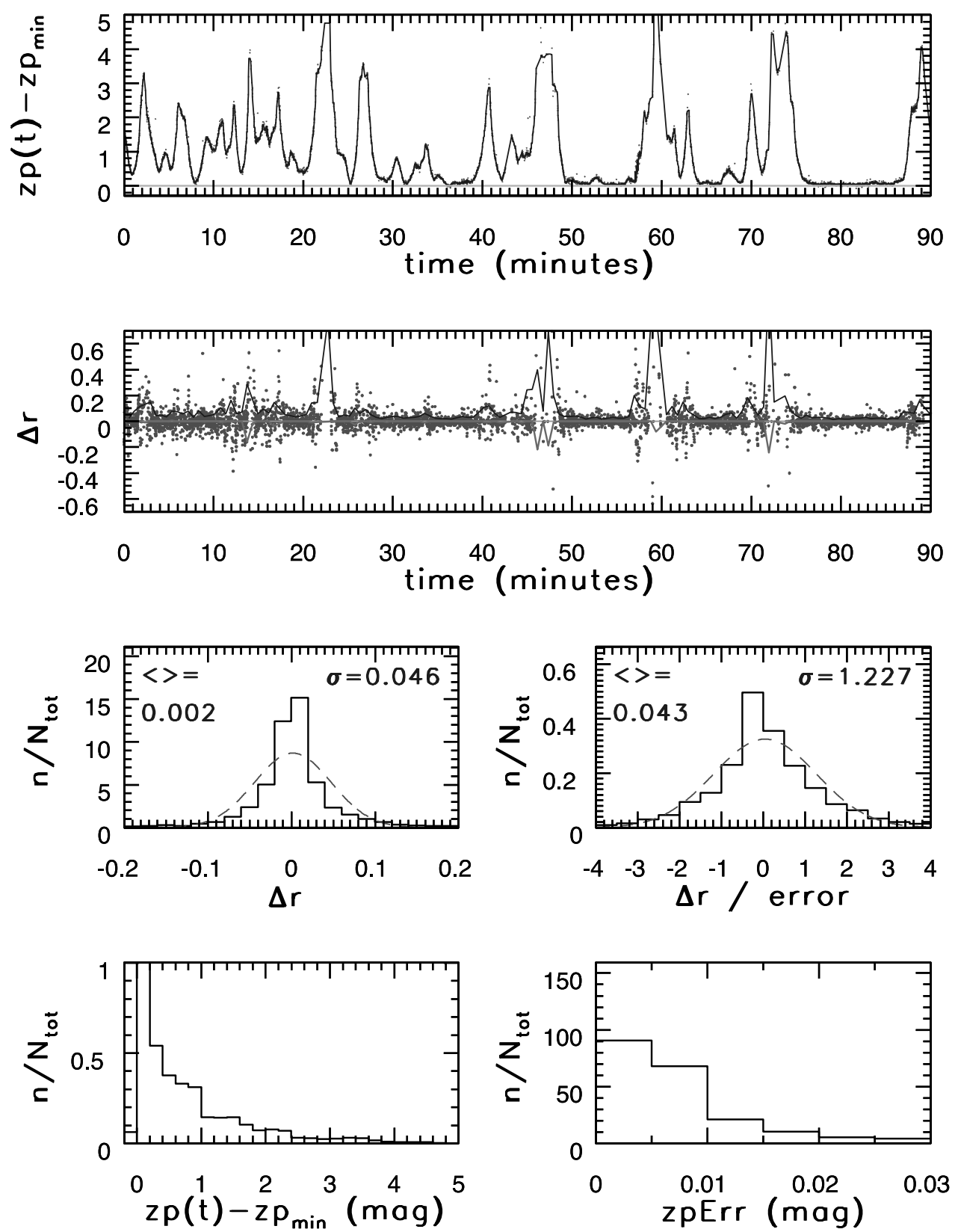

FIG. 15.-Top panel: Summary of the behavior of cloud extinction in the $r$ band over $1.5 \mathrm{hr}$ during SDSS-II run 5646. Individual calibration stars are shown by dots, and the adopted zero point is shown by the line. Second panel: Calibration residuals for each star (dots). The rms scatter for these residuals evaluated for each field is shown by the line. Third row of panels: Distribution of the residuals is shown in the left panel (solid line). The median and equivalent Gaussian $\sigma$ evaluated from the interquartile range are also shown in this panel, as well as a Gaussian corresponding to these parameters (dashed line). The right panel is analogous, except that the residuals are normalized by the expected errors. Bottom panels: Distribution of implied cloud extinction is shown in the left panel, and the distribution of standard errors for the adopted photometric zero points (computed from the rms width of the distribution of residuals) is shown in the right panel (a few points, about 4 out of 1800 calibration patches, are off scale). [See the electronic edition of the Journal for a color version of this figure.]

\subsubsection{The Cloud Color and Structure Function}

We detect no dependence of the calibration residuals on the stellar color or cloud thickness at the level of a few millimagnitudes. This is consistent with the lack of selective extinction by clouds. The lack of a color correlation in the $u$ and $z$ bands implies that the well-known cloud grayness extends beyond visual wavelengths. Another method to quantify selective extinction by clouds is to directly compare zero points from different bands. As shown in Figure 20, the cloud extinction is similar in all bands for most fields. A few cases where there are deviations of a few tenths of a magnitude can be easily understood as due to temporal changes in the cloud opacity (recall that the data from different bands are obtained over $\sim 5$ minutes of time).

The calibration accuracy is determined by the size of the calibration patches. For example, a smaller patch would suffer less from the spatial variation of cloud extinction, but it would not have enough stars to beat down the noise of their individual photometric measurements ( $\sim 0.02$ mag for sufficiently bright stars). The detailed scaling of this accuracy with patch size depends on the cloud spatial SF. The geometry of the SDSS camera allows us to study the cloud SF on scales exceeding $2^{\circ}$. Figure 21 compares zero points in different columns for two runs with significantly different cloud behavior. While zero points from different columns 

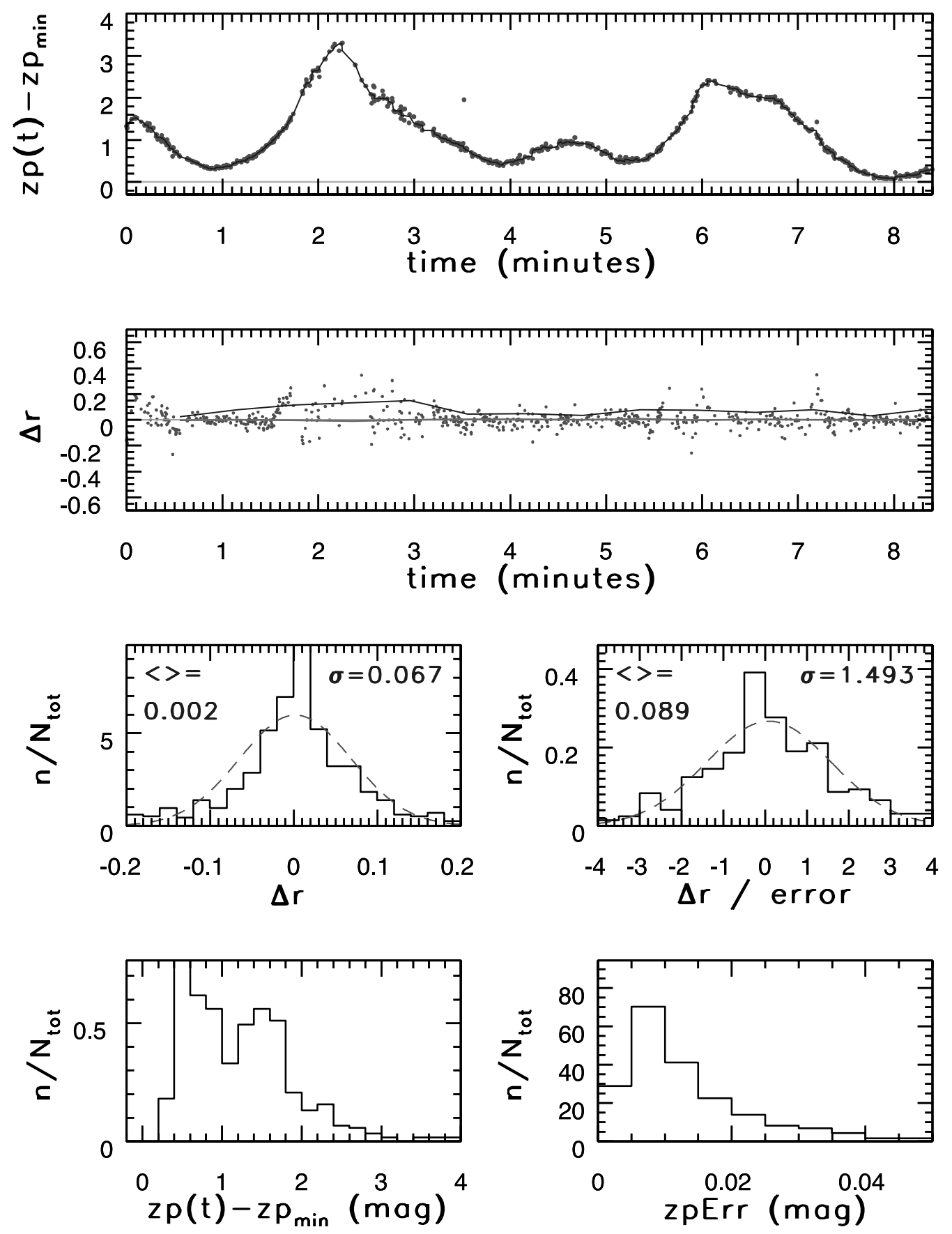

Fig. 16. - Same as Fig. 15, but showing only $\sim 8$ minutes of data with large cloud extinction. Note that the changes in cloud extinction are resolved down to timescales well below 1 minute. [See the electronic edition of the Journal for a color version of this figure.]

generally track each other, there can be differences exceeding a magnitude (they generally scale with the cloud optical thickness). These differences increase with the distance between the camera columns. Figure 22 shows typical behavior: for small spatial scales $\left(<2^{\circ}\right)$ the SF is roughly a linear function of distance, and it scales roughly linearly with the cloud extinction. At a $1^{\circ}$ scale, the $S F$ is typically of order $2 \%-10 \%$ of the cloud extinction. For example, even for clouds 3 mag thick, the SF at $2^{\prime}$ scales is typically $<0.01 \mathrm{mag}$.

\subsubsection{Implications for Surveys Such as LSST}

The LSST is a proposed imaging survey that will attempt to maximize its observing time by accepting nonphotometric condi- tions. At the same time, it has adopted exquisite requirements for its photometric accuracy, including 1\% accuracy of its internal photometric zero-point errors across the sky. Our analysis allows us to answer the following question: What is the largest cloud extinction that still allows photometric calibration accurate to $1 \%$ ?

A similar approach to the calibration of LSST data as presented here (assuming that a standard star catalog is available, e.g., from prior demonstrably photometric nights) would benefit from several effects:

1. The LSST will not use a drift-scanning technique, and thus the calibration patches can be squares; for the same area, this results in an $\sim 5$ times smaller angular scale $\left(\sim 3^{\prime}\right)$, compared to the 

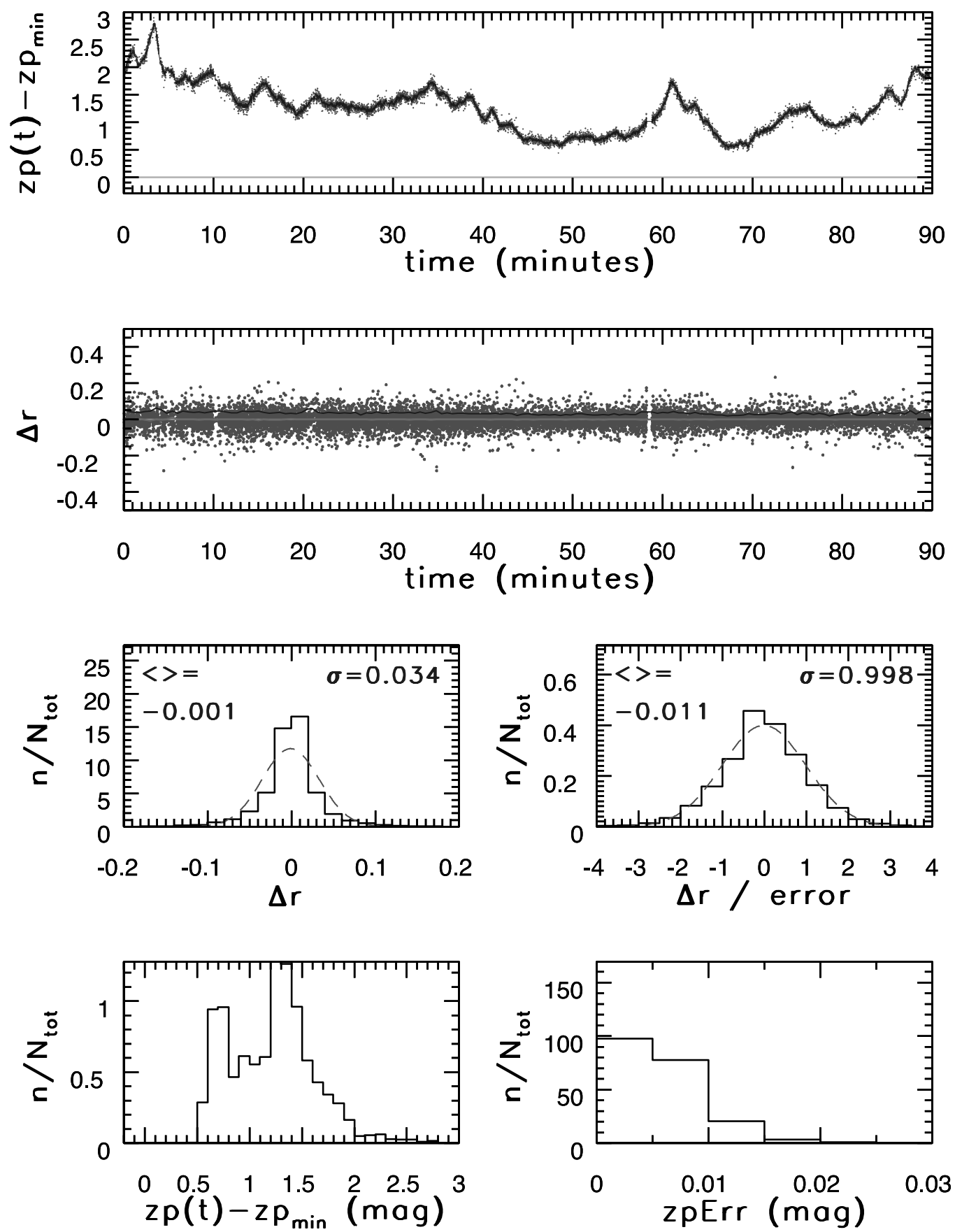

Fig. 17. - Same as Fig. 15, but showing $1.5 \mathrm{hr}$ of data from run 5759, which had somewhat thinner and much more stable cloud cover. Note that the median photometric zero-point error is below $0.01 \mathrm{mag}$, although the median cloud extinction is larger than $1 \mathrm{mag}$. [See the electronic edition of the Journal for a color version of this figure.]

1:20 rectangles we have used to calibrate SDSS drift-scanning data. On these angular scales, the cloud SF is roughly linear, and thus the zero-point error is $\sim 5$ times smaller, or of the order of $1 \%$ or less through clouds as thick as 1 mag (conservatively assuming that SDSS errors would be $0.05 X$; see $\S 3.1 .3)$. We note that the shorter exposure time for LSST $(30 \mathrm{~s}$, or about a factor of 2 shorter than for SDSS) is not relevant because clouds would typically move by more than a degree during the exposure. This is a distance more than an order of magnitude larger than the size of the calibration patch, and thus the SF analysis remains valid.

2. LSST data will be deeper than that of SDSS by about 23 mag. With a conservative assumption that $\log N \propto 0.3$ mag for faint stars (0.6 for Euclidean counts), the surface density of the calibration stars will be about 10 times larger for LSST than for
SDSS. This larger density enables 10 times smaller patches, or about 3 times smaller angular scale for calibration $\left(\sim 1^{\prime}\right)$, resulting in another factor of 3 improvement of accuracy.

3. Fitting a smooth function for cloud opacity over several calibration patches would result in further improvements.

The first two points predict that LSST data could be calibrated with the required 1\% accuracy even through 3 mag thick clouds. Given the various extrapolations, we conservatively suggest the range of 1-3 mag as the upper limit on the acceptable cloud opacity. $^{46}$

\footnotetext{
${ }^{46}$ Of course, cloud opacity decreases the imaging depth, and data with clouds thicker than $\sim 1$ mag may be undesirable for reasons other than calibration accuracy.
} 
Run 5759, u band, column 1
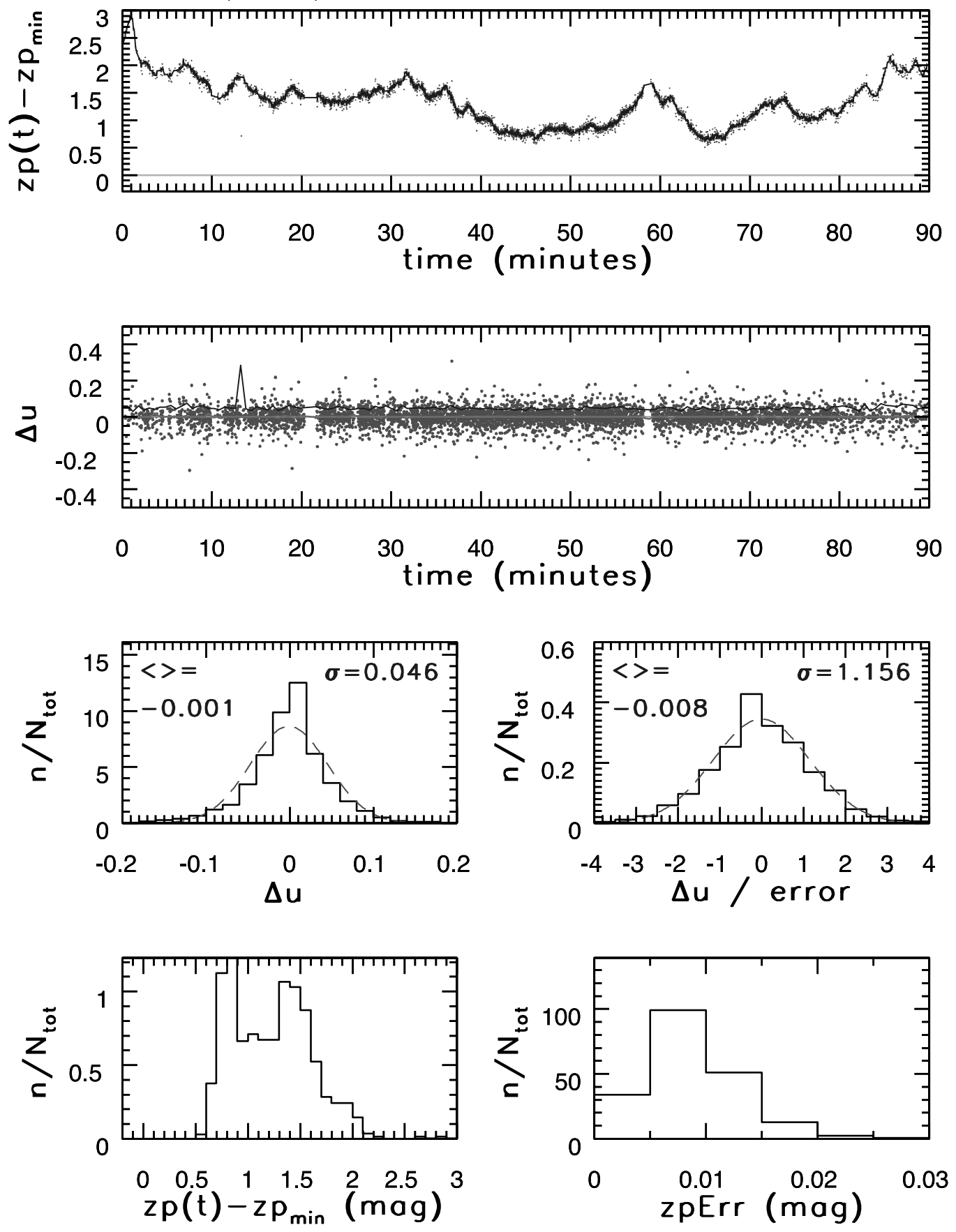

FIG. 18. - Same as Fig. 17, but showing the $u$-band calibration summary. Despite the smaller number of calibration stars than in redder bands, and over a magnitude of cloud extinction, it is still possible to photometrically calibrate these data with a median error of only $\sim 0.01 \mathrm{mag}$. [See the electronic edition of the Journal for a color version of this figure.]

\subsection{The Morphology of the Stellar Locus}

The improved accuracy of averaged photometry provides "crisper" color-color diagrams and also reveals new morphological features. An example of such a color-color diagram is shown in Figure 23.

This is a similar plot to that of Figure 1 of Smolčić et al. (2004), except that only nonvariable point sources are shown (note the absence of quasars) and averaged photometry is used. The white dwarf/M dwarf "bridge" discussed by Smolčić et al. is clearly visible, as well as the locus of probable solar metallicity giants (this identification is based on models; e.g., Kurucz 1979), which deviates from the main locus at $u-g \sim 2.5$ and $g-r \sim 1$. Note also the well-defined blue horizontal-branch locus $(u-g \sim 1.1$ and $g-r$ from -0.3 to 0.1$)$ and the white dwarf locus $(u-g \sim$ 0.35 and $g-r$ from -0.3 to $\sim 0.0$ ). A new locus-like feature that is not visible in Figure 1 of Smolčić et al. is discernible at $u-$ $g \sim 0$ and $g-r \sim-0.2$. The great value of the accurate $u$-band photometry is clearly evident at, e.g., $g-r=-0.2$; the $u-g$ color distribution is trimodal! The bluest branch is consistent with He white dwarfs and the middle branch with hydrogen white dwarfs, as supported by Bergeron et al. (1995) white dwarf models 

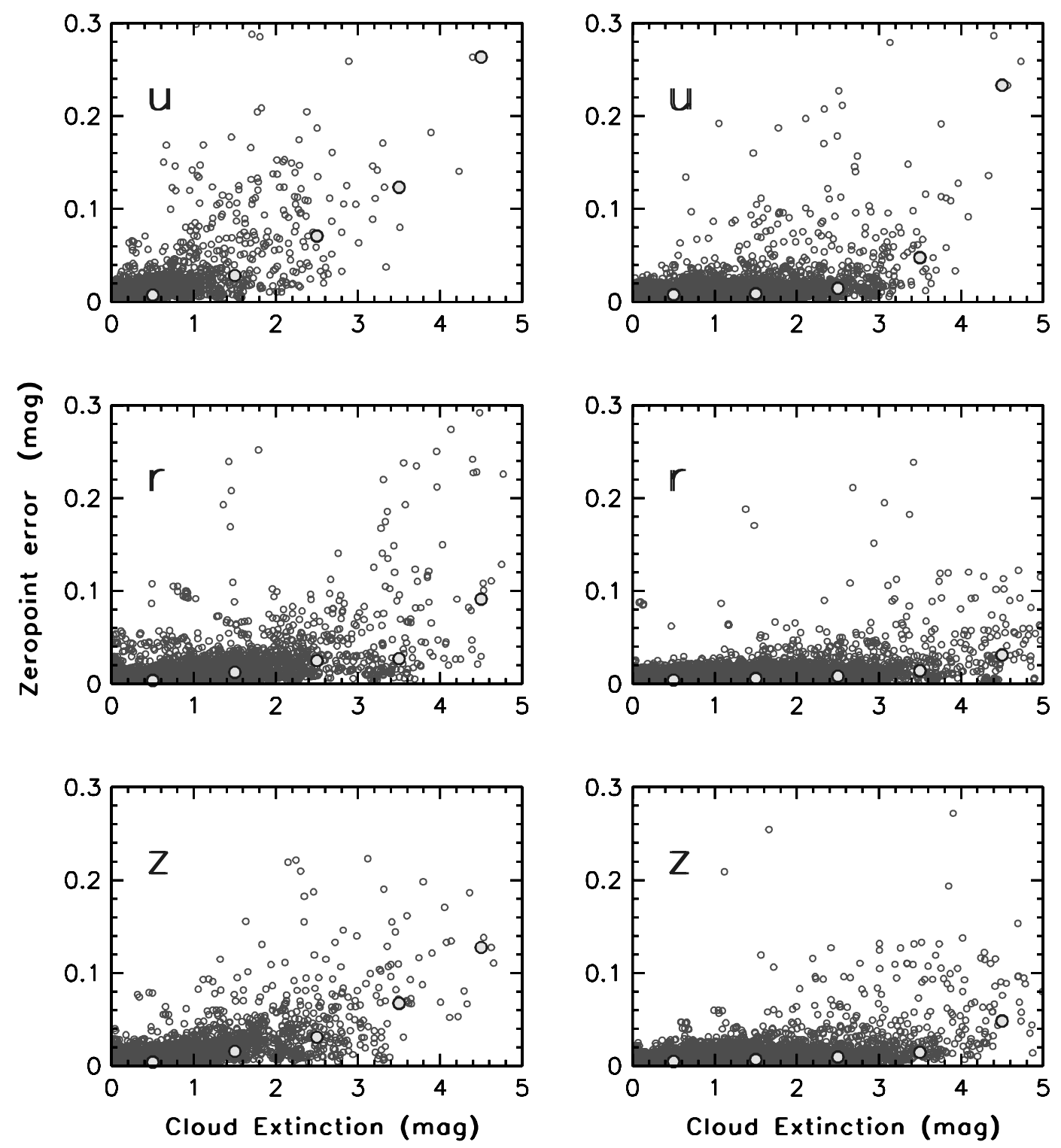

FIG. 19.- Summary of calibration accuracy as a function of cloud extinction and band ( $u r z$, as marked in the panels). Each small symbol represents one calibration patch (an $\sim 9 \operatorname{arcmin}^{2}$ large rectangle with a 1:20 aspect ratio). The zero-point error is determined from the rms of the photometric residuals. The large symbols show the median zero-point error in $1 \mathrm{mag}$ wide bins of cloud extinction. The left column shows data for one of the photometrically worst SDSS-II supernova runs (5646), and the right column shows data for a run with optically thick but exceptionally smooth clouds (5759). Note that the data can be calibrated with zero-point errors typically smaller than a few percent even through clouds several magnitudes thick. [See the electronic edition of the Journal for a color version of this figure.]

and detailed analysis of SDSS spectra (Eisenstein et al. 2006). The reddest branch is made of blue horizontal-branch stars (see Sirko et al. 2004 and references therein).

The exciting fact that one can distinguish $\mathrm{He}$ and $H$ white dwarfs using photometry alone is a consequence of the improved photometric accuracy due to averaging many epochs. Figure 24 reiterates that point. Note the striking difference between the two bottom panels: while one could be convinced that the He white dwarf sequence is a real feature in the bottom right panel, its existence is clearly evident when using the improved photometry, as shown in the bottom left panel. In summary, the multiepoch observations provide both the identification of variable sources and much more accurate colors for nonvariable sources. This bodes well for science deliverables from upcoming large-scale imaging surveys. For example, LSST will obtain over its $10 \mathrm{yr}$ mission repeat imaging similar to that discussed here, but about 2.5 mag deeper, with about 100 or more observations per band and object and over an area about 2 orders of magnitude larger. Although these new surveys will not have a spectroscopic component like SDSS did, the multiepoch nature of their imaging will provide alternative information-rich data sets.

\section{DISCUSSION AND CONCLUSIONS}

Using repeated SDSS measurements, we have constructed a catalog of over 1 million candidate standard stars. The catalog is publicly available from the SDSS Web site. ${ }^{47}$ Several independent tests suggest that both internal zero-point errors and random photometric errors for stars brighter than 19.5, 20.5, 20.5, 20, and 18.5 in ugriz, respectively, are at or below 0.01 mag (about 2-3 times better than for an individual SDSS scan). This is by far the largest existing catalog with multiband optical photometry

\footnotetext{
${ }^{47}$ See http://www.sdss.org/dr5/products/value_added/index.html.
} 
Run 5646, column 1, implied cloud color
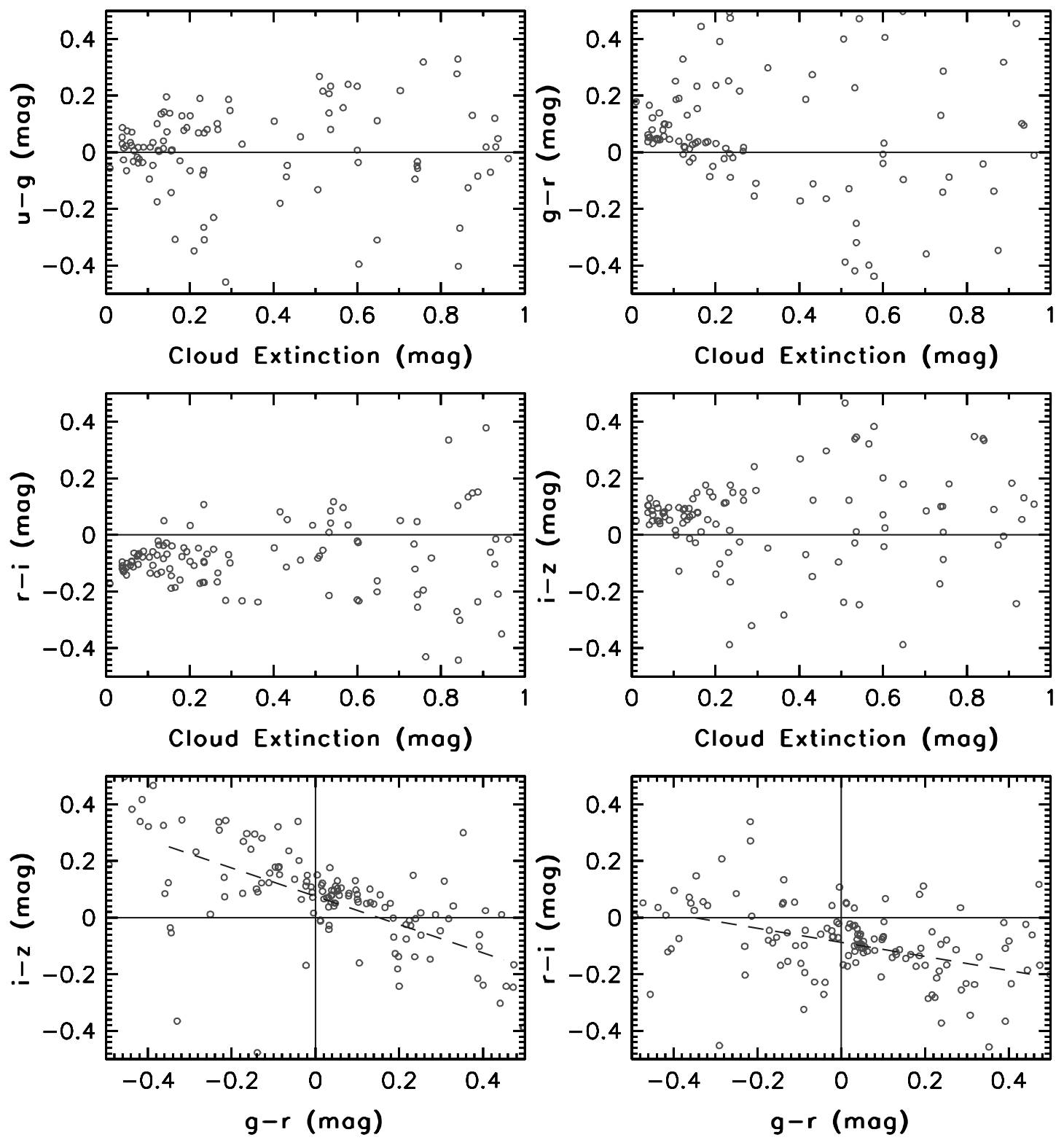

FIG. 20.-Color of cloud extinction in SDSS bands. Each symbol represents one field and shows the difference in cloud extinction between the two bands as a function of the $r$-band extinction. The measurements in different bands are obtained over $\sim 5$ minutes of time, and thus even gray clouds with spatially varying extinction could produce the observed nongray (nonzero) values. The dashed lines in the bottom two panels indicate the expected correlation if the color variations are due to temporal changes in the gray cloud thickness (rather than due to intrinsic color changes). [See the electronic edition of the Journal for a color version of this figure.] 

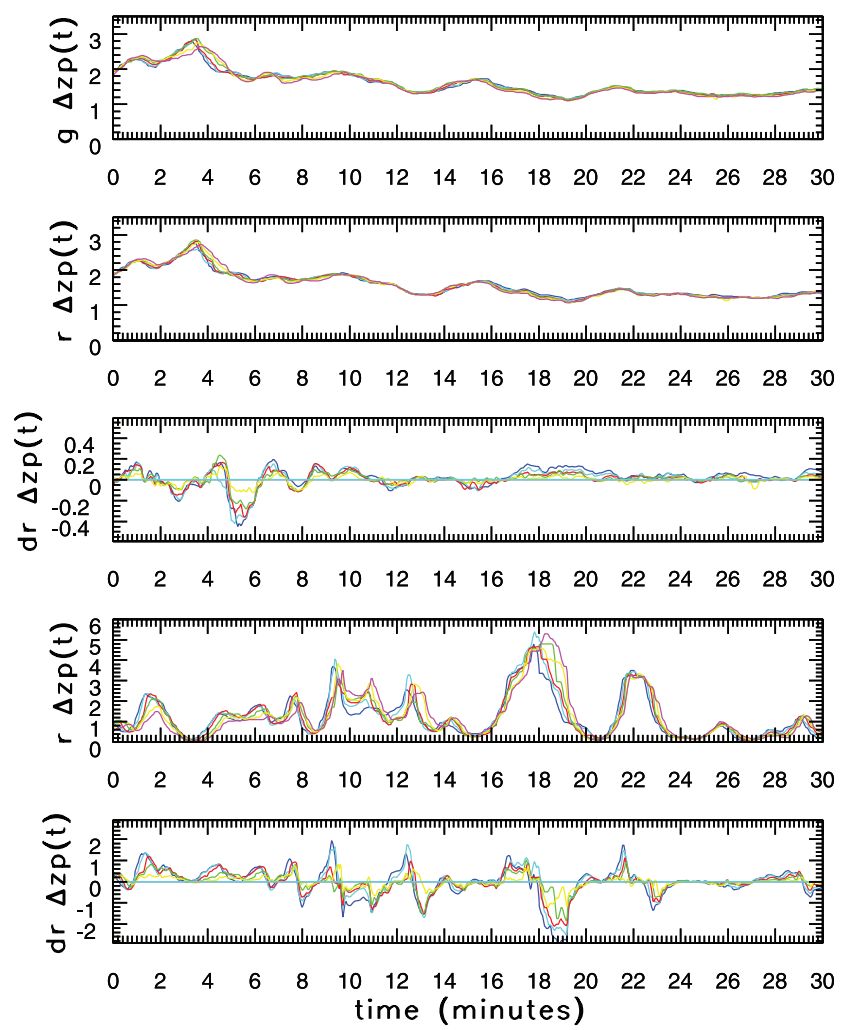

FIG. 21.-Comparison of cloud extinction independently measured for six camera columns. Top two panels: 30 minutes of measurements in the $g$ and $r$ bands for the same run (5759) shown in Fig. 17. Individual camera columns are colorcoded according to the legend shown in Fig. 6 (top). Third panel: Difference between the $r$-band zero points measured in one of the edge columns (6) and zero points from the other five columns. Bottom two panels: Same as second and third panels, but with data from a run with exceptionally patchy clouds (5646; the first 30 minutes of the data from Fig. 15 are shown). Note the varying scale for the $y$-axis.

accurate to $\sim 1 \%$ and breaks the accuracy barrier discussed by, e.g., Stubbs \& Tonry (2006 and references therein; see also Maíz Apellániz [2006] for a discussion of $1 \%$ photometry in other photometric systems). These observations were not obtained for the specific purpose of calibration but were part of the regular SDSS observational program. When compared to, for example, the heroic calibration efforts by A. U. Landolt, P. Stetson, and others, it seems justified to call the method presented here "industrial" photometry. However, the catalog presented here is not without its problems.

The selection of candidate stars was simply based on the absence of variability. It is fairly certain that not all variable sources are recognized because of the limited number of repeated observations $(\sim 10)$. For example, an eclipsing binary with a much shorter eclipse duration than the orbital period could easily escape detection. Furthermore, some of these sources may not even be stars. A cross-correlation with the SDSS spectroscopic database yields 99,000 matches in the overlapping region, including 44,000 spectra classified as stars. About 70 candidate standard stars are actually spectroscopically confirmed quasars! Apparently, a small fraction of quasars (a few percent; for a detailed analysis, see B. Sesar et al. 2007, in preparation) cannot be detected by variability (at least not using the number of epochs, their time distribution, and the photometric accuracy employed in this work). Indeed, we have also found three spectroscopically confirmed SDSS quasars among Stetson's standards which were observed 20-30 times and which showed no variation. Similarly, about 300 candidate standard stars have SDSS spectra classified as galaxies. Nevertheless, the inspection of color-color diagrams

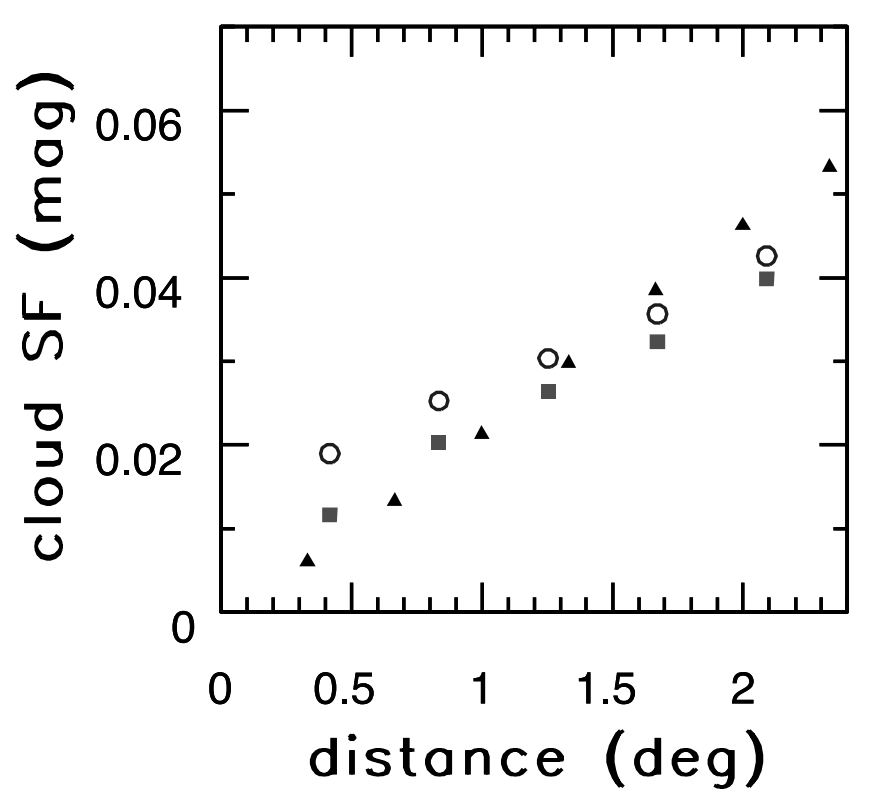

FIG. 22.-Cloud SF in the $r$ band for run 5759 and for the same stretch of data as shown in Fig. 17 (the median cloud extinction is $1.3 \mathrm{mag}$ ). The circles show the rms width of the distribution of zero-point differences between camera column 1 and the five other columns. This width is corrected for a 0.015 mag contribution from the measurement errors and shown by squares. The triangles show the width of the distribution of zero-point differences in the in-scan direction, with the distance scale multiplied by 30 . This multiplication factor measures the cloud speed relative to the boresight in the in-scan direction (see $\S 3.1 .1$ ). [See the electronic edition of the Journal for a color version of this figure.]

strongly suggests that the overwhelming majority of the standard stars are found on the stellar locus.

Remaining systematic errors are another important concern. Effectively, we have assumed that PT problems average out in many patches when deriving flat-field corrections using stellar colors. This may not be true at a level not much smaller than $1 \%$, and thus the remaining gray problems at such a level may be present in the catalog. Despite these residual problems, we believe that

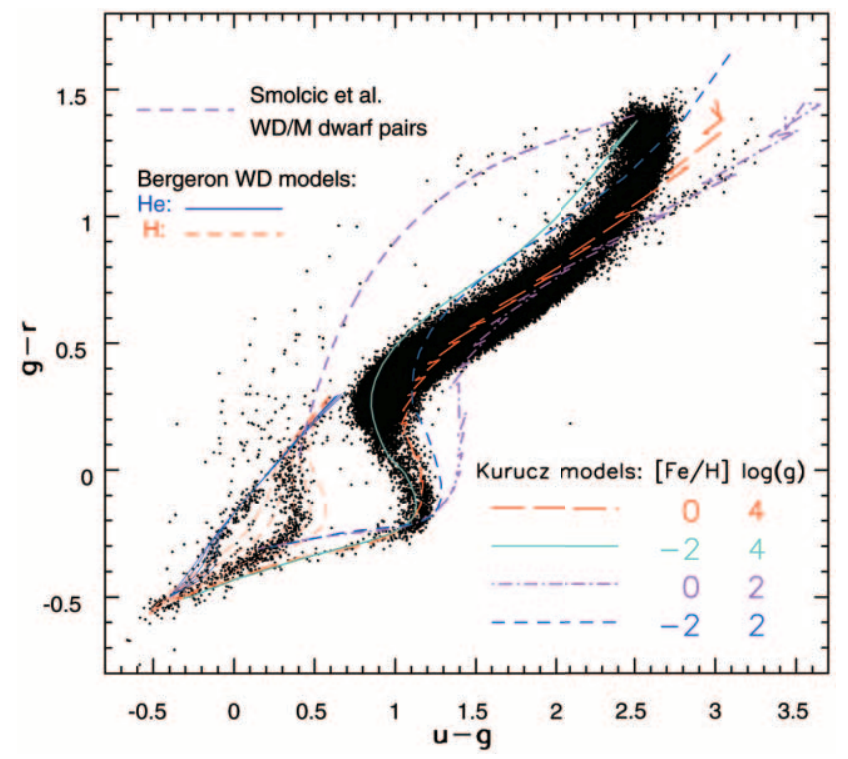

FIG. 23.-The $g-r$ vs. $u-g$ color-color diagrams for all nonvariable point sources constructed with the improved averaged photometry (dots). Various stellar models (Kurucz 1979; Bergeron et al. 1995; Smolčić et al. 2004) are shown by lines, as indicated in the figure. 

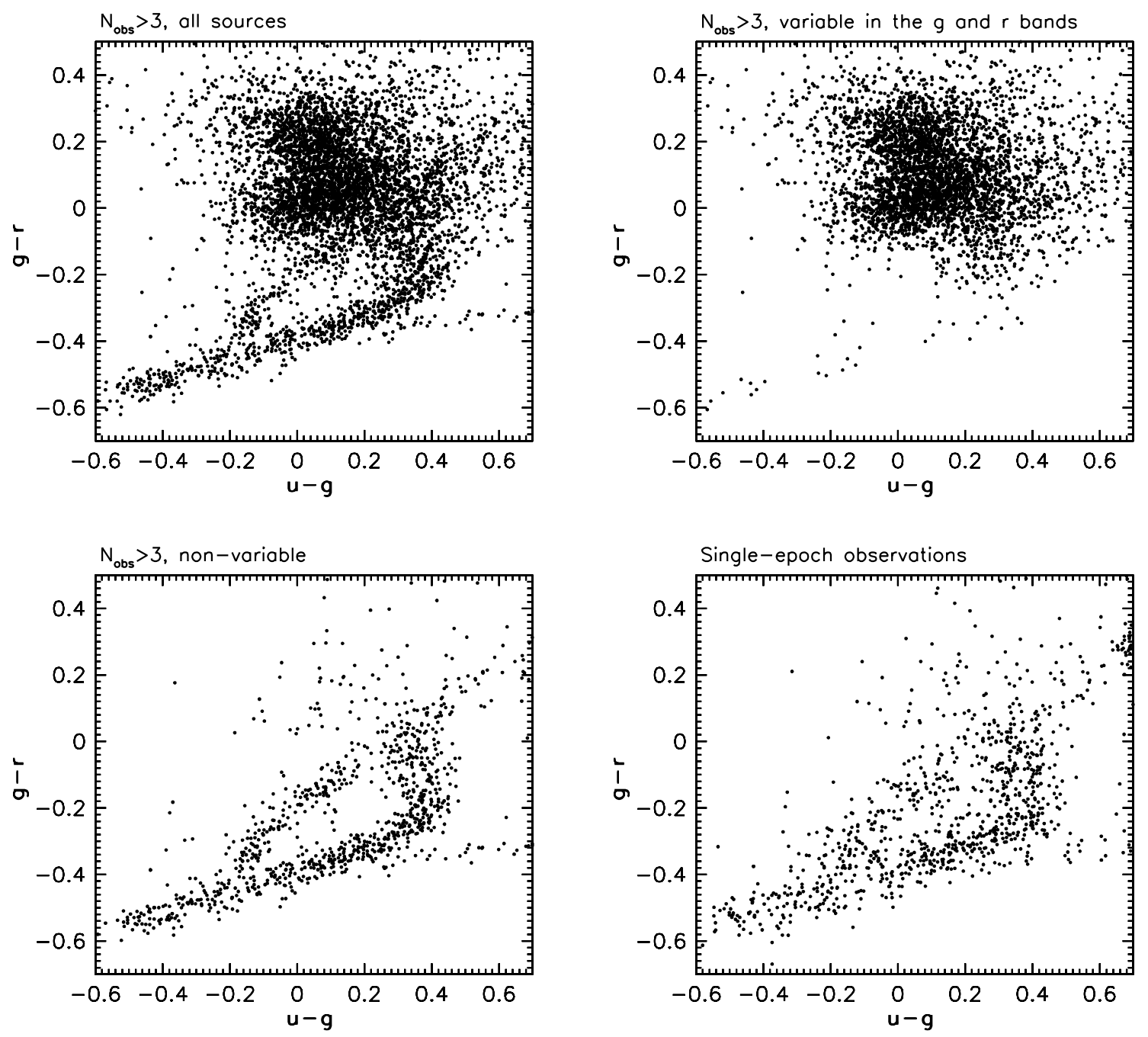

FIG. 24.-Advantages of repeated photometric measurements. Top left panel: Blue corner of the $g-r$ vs. $u-g$ diagram from Fig. 23 for all point sources with the averaged photometry. Top right panel: Only the variable sources (dominated by low-redshift quasars). Bottom left panel: Nonvariable sources (dominated by white dwarfs) classified using low-order light-curve moments. Bottom right panel: Same nonvariable sources, but using their DR5 single-epoch photometry. A comparison of the bottom two panels shows the striking improvement made possible by the use of multiple observations of the same field.

internal consistency of the catalog (i.e., when ignoring $\Delta_{m}$ from eq. [4]) is such that the rms width for the function $\delta_{m}$ (R.A., decl.) from equation (4) evaluated for all stars in the catalog is at most $0.01 \mathrm{mag}$ in the griz bands and perhaps just slightly larger in the $u$ band (very unlikely exceeding $0.02 \mathrm{mag}$ ). In addition to gray problems and overall flat-field errors, the dependence of flat fields on source color is probably the largest remaining systematic error.

We illustrate several uses for this catalog, including the calibration of highly nonphotometric data and robust selection of stars with peculiar colors. We find that LSST and similar surveys will be able to observe in partially cloudy (nonphotometric) nights because even cloudy data can be accurately calibrated with a sufficiently dense network of calibration stars. Such a dense network will be self-calibrated by LSST very soon after first light, using an approach developed for SDSS data by Padmanabhan et al. (2007). Given such a network, SDSS experience suggests that LSST can maintain its required photometric calibration accuracy of $1 \%$ even when observing through 1-3 mag thick clouds.

Perhaps the most exciting conclusion of this work is that it may become obsolete in only a few years due to the advent of nextgeneration surveys such as Pan-STARRS and LSST.
We are thankful to Peter Stetson for his most useful comments and for providing his latest standard star photometry to us. Ž. I. and B. S. acknowledge support by NSF grant AST 05-51161 to LSST for design and development activity, and H. M. acknowledges support by NSF grant AST 06-07518. We are grateful to the referee, Jesús Maíz Apellániz, for his careful reading and excellent comments.

Funding for the SDSS and SDSS-II has been provided by the Alfred P. Sloan Foundation, the Participating Institutions, the National Science Foundation, the US Department of Energy, the National Aeronautics and Space Administration, the Japanese Monbukagakusho, the Max Planck Society, and the Higher Education Funding Council for England. The SDSS Web site is http://www.sdss.org.

The SDSS is managed by the Astrophysical Research Consortium for the Participating Institutions. The Participating Institutions are the American Museum of Natural History, the Astrophysical Institute Potsdam, the University of Basel, the University of Cambridge, Case Western Reserve University, the University of Chicago, Drexel University, Fermilab, the Institute for Advanced Study, the Japan Participation Group, Johns Hopkins University, 
the Joint Institute for Nuclear Astrophysics, the Kavli Institute for Particle Astrophysics and Cosmology, the Korean Scientist Group, the Chinese Academy of Sciences, Los Alamos National Laboratory, the Max Planck Institute for Astronomy, the Max Planck
Institute for Astrophysics, New Mexico State University, Ohio State University, the University of Pittsburgh, the University of Portsmouth, Princeton University, the United States Naval Observatory, and the University of Washington.
Abazajian, K., et al. 2003, AJ, 126, 2081

. 2004, AJ, 128, 502 2005, AJ, 129, 1755

Adelman-McCarthy, J. K., et al. 2006, ApJS, 162, 38

Baldry, I. K, Glazebrook, K., Brinkmann, J., Ivezić, Ž., Lupton, R. H., Nichol, R. C., \& Szalay, A. S. 2004, ApJ, 600, 681

Bergeron, P., Saumon, D., \& Wesemael, F. 1995, ApJ, 443, 764

Eisenstein, D. J., et al. 2001, AJ, 122, 2267 . 2006, ApJS, 167, 40

Fan, X. 1999, AJ, 117, 2528

Finlator, K., et al. 2000, AJ, 120, 2615

Flaugher, B., et al. 2006, BAAS, 209, 2201

Fukugita, M., Ichikawa, T., Gunn, J. E., Doi, M., Shimasaku, K., \& Schneider, D. P. 1996 , AJ, 111, 1748

Gunn, J. E., \& Stryker, L. L. 1983, ApJS, 52, 121

Gunn, J. E., et al. 1998, AJ, 116, 3040 2006, AJ, 131, 2332

Helmi, A., et al. 2003, ApJ, 586, 195

Hogg, D. W., Finkbeiner, D. P., Schlegel, D. J., \& Gunn, J. E. 2001, AJ, 122, 2129

Holberg, J. B., \& Bergeron, P. 2006, AJ, 132, 1221

Ivezić, Ž., Vivas, A. K., Lupton, R. H., \& Zinn, R. 2005, AJ, 129, 1096

Ivezić, Ž., et al. 2003, Mem. Soc. Astron. Italiana, 74, 978 . 2004a, Astron. Nachr., 325, 583

- 2004b, in ASP Conf. Ser. 311, AGN Physics with the Sloan Digital Sky Survey, ed. G. T. Richards \& P. B. Hall (San Francisco: ASP), 437 2006, Mem. Soc. Astron. Italiana, 77, 1057

. 2007, in ASP Conf. Ser. 364, The Future of Photometric, Spectrophotometric, and Polarimetric Standardization, ed. C. Sterken (San Francisco: ASP), 165

Jurić, M., et al. 2005, ApJ, submitted (astro-ph/0510520)

Kaiser, N., et al. 2002, Proc. SPIE, 4836, 154

Kurucz, R. L. 1979, ApJS, 40, 1

\section{REFERENCES}

Landolt, A. U. 1983, AJ, 88, 439 1992, AJ, 104, 340

Lenz, D. D., Newberg, J., Rosner, R., Richards, G. T., \& Stoughton, C. 1998, ApJS, 119, 121

Lupton, R. H. 1993, Statistics in Theory and Practice (Princeton: Princeton Univ. Press)

Lupton, R. H., Gunn, J. E., \& Szalay, A. 1999, AJ, 118, 1406

Lupton, R. H., Ivezić, Ž., Gunn, J. E., Knapp, G. R., Strauss, M. A., \& Yasuda, N. 2002, Proc. SPIE, 4836, 350

Maíz Apellániz, J. 2006, AJ, 131, 1184

Oke, J. B., \& Gunn, J. E. 1983, ApJ, 266, 713

Padmanabhan, N., et al. 2007, ApJ, submitted (astro-ph/0703454)

Pier, J. R., Munn, J. A., Hindsley, R. B., Hennesy, G. S., Kent, S. M., Lupton, R. H., \& Ivezić, Ž. 2003, AJ, 125, 1559

Sako, M., et al. 2005, BAAS, 37, 1408

Schlegel, D., Finkbeiner, D. P., \& Davis, M. 1998, ApJ, 500, 525 (SFD98)

Scranton, R., Connolly, A. J., Szalay, A. S., Lupton, R. H., Johnston, D., Budavari, T., Brinkman, J., \& Fukugita, M. 2005, preprint (astro-ph/0508564)

Scranton, R., et al. 2002, ApJ, 579, 48

Sesar, B., et al. 2006, AJ, 131, 2801

Sirko, E., et al. 2004, AJ, 127, 899

Smith, J. A., et al. 2002, AJ, 123, 2121

Smolčić, V., et al. 2004, ApJ, 615, L141

Stetson, P. B. 2000, PASP, 112, 925 2005, PASP, 117, 563

Stoughton, C., et al. 2002, AJ, 123, 485

Strateva, I., et al. 2001, AJ, 122, 1861

Stubbs, C. W., \& Tonry, J. L. 2006, ApJ, 646, 1436

Tucker, D. L., et al. 2006, Astron. Nachr., 327, 821

Tyson, J. A. 2002, Proc. SPIE, 4836, 10

Yasuda, N., et al. 2001, AJ, 122, 1104

York, D. G., et al. 2000, AJ, 120, 1579 